\title{
An asymptotic expansion-based method for a spectral approach in equivalent statistical linearization
}

\author{
T. Canor ${ }^{\mathrm{a}, \mathrm{b}, *}$, N. Blaise ${ }^{\mathrm{a}}$, V. Denoël ${ }^{\mathrm{a}}$ \\ ${ }^{a}$ University of Liège, Department of Architecture, Geology, Environment and Constructions, Structural Engineering Division, \\ Chemin des Chevreuils, 1, B52/3, 4000 Liège, Belgium \\ ${ }^{b}$ F.R.S.-FNRS, National Fund for Scientific Research, 1000 Bruxelles, Belgium
}

\begin{abstract}
Equivalent linearization consists in replacing a nonlinear system with an equivalent linear one whose parameters are tuned with regard to the minimization of a suitable function. In particular, the Gaussian equivalent linearization expresses the properties of an equivalent linear system in terms of the mean vector and the covariance matrix of the responses, which are the unknowns of the optimization problem in a spectral approach. Even though the system has been linearized, the resulting set of equations is nonlinear. The computational effort in this method pertains to the solution of a possibly large set of nonlinear algebraic equations involving integrals and inversions of full matrices. This work proposes to develop and apply an asymptotic expansion-based method to facilitate and to improve the statistical linearization for large nonlinear structures. The proposed developments demonstrate that for slightly to moderately coupled nonlinear systems, the equivalent linearization can be applied with an appropriate modal approach and eventually seen as a convergent series initiated with the stochastic response of a main decoupled linear system. With this method, the computational effort is attractively reduced, the conditioning of the set of nonlinear algebraic equations is improved and inversion of full transfer matrices and repeated integrations are avoided. The paper gives a formal description of the method and illustrates its implementation and performances with the computation of stationary responses of nonlinear structures subject to coherent random excitation fields.
\end{abstract}

Keywords: equivalent linearization, stochastic modal analysis, asymptotic expansion, wind engineering, earthquake engineering, nonlinear dynamics

$P A C S: \mathrm{xxx} \cdot \mathrm{xxx}, \mathrm{xxx} \cdot \mathrm{xxx}, \mathrm{xxx} \cdot \mathrm{xxx}$

2008 MSC: $\mathrm{xxx} \cdot \mathrm{xxx}$

\section{Introduction}

A classical result from probabilistic theory states that linear deterministic systems driven by Gaussian processes respond with Gaussian processes. The joint probability density function of the responses is thus completely characterized by a mean vector and a covariance matrix. However, for nonlinear systems or in the case of non-Gaussian excitations, the computation of the response of the system is more complicated, partly due to the statistical polymorphism of a non-Gaussian process.

Discarding straightaway the Fokker-Planck equation due to the curse of dimensionality [1, 2], the Monte Carlo approach is considered as the only tractable method to compute the non-Gaussian response of large-dimensional nonlinear systems [3, 4. Briefly, the method consists in generating samples of the excitation to compute samples of the system response by means of deterministic solvers. Although the simulation-based framework is extensively used in risk analysis and risk quantification [5], the computational burden remains a major drawback of this method. Indeed, the generation of random samples from coherent random fields, as wind acting on large structures [6, 7, may be prohibitive. Therefore, the use of approximate methods is attractive, especially in a design stage or in an optimization procedure involving many parameters and repeated operations.

Many approximate methods have been developed for decades : the averaging method [8, the equivalent linearization [9, 10, quadratization and cubicization [11, non-Gaussian closures [12] are seemingly the most famous. Among them, the equivalent linearization, originally introduced by Botoon and Caughey [13, 14 can be used for the analysis of high-dimensional nonlinear structures, as encountered in earthquake engineering [15, 16, 17] or in

\footnotetext{
${ }^{*}$ Corresponding author

Email addresses: t.canor@ulg.ac.be (T. Canor), n.blaise@ulg.ac.be (N. Blaise), v.denoel@ulg.ac.be (V. Denoël)
} 
wind engineering [18, 19, 20]. The main idea of the equivalent linearization consists in replacing the nonlinear system with an equivalent linear one by minimizing an error criterion depending on the parameters of the equivalent system. Though different criteria have been proposed [9, 21, the most robust and advantageous one remains the minimization of the mean squared discrepancy, by tuning the parameters of the equivalent system, especially as the excitations are diffusive processes.

The equivalent linearization method benefit from valuable features of linear systems. First, the modal projection can be used to reduce the size of the equation of motion. Secondly, it is more convenient to work in the frequency domain for stationary processes, while realistic loadings are usually modeled by large Power Spectral Density (PSD) matrices, as it is the case for coherent wind or seismic fields. Finally, the input-output Gaussianity is preserved. Consequently, the equivalent linearization may compete with Monte Carlo simulation in the estimation of the first two statistical moments.

In the linearization method, assumptions on the statistical distribution of the response is formulated : the Gaussian Equivalent Linearization (GEL) supposes that the responses of the system are Gaussian processes, but statistical linearization methods have been extended to non-Gaussian processes [22, 23, 24] with limited success. The GEL expresses the properties of the equivalent linear system in terms of the mean vector and the covariance matrix of the response of the system. Even though the system has been linearized, the set of equations to calculate the covariance matrix of the system is nonlinear. The computational effort in this method pertains to the solution of a possibly large set of nonlinear algebraic equations, all the more for large nonlinear structures.

This work proposes to develop and apply a perturbation approach, as formerly investigated by the authors for deterministic [25] and stochastic [26] linear systems, to facilitate and to improve the GEL of a nonlinear structure subject to stationary loadings. Our approach exposed in a stationary setting can be extended to some classes of evolutionary problems [27, 28] (alternative to fully-nonstationary excitations [29]), provided the quasi-stationary assumption is justified, i.e. the natural period of the structure is small compared to the duration of the evolutionary random excitation [30, 31.

Since many optimization algorithms can be used to solve the nonlinear equation set inherent to statistical linearization, the development of a solver accounting for the specificities of this set has not often been addressed by the research community. Provided the excitation can be modeled by filtered white noises, the covariance matrix of the response is expressed by a Lyapunov equation. This equation can be solved in particular by direct algorithm [32, 33, 34. Nonetheless, if the excitation is modeled as a coherent field in the frequency domain, the Itô procedure cannot be applied. In the context of equivalent linearization with a spectral approach, a fixed-point algorithm is a convenient and readily implemented method 10. However, this algorithm behaves poorly in terms of convergence [35, especially for large equation sets. Consequently, a gradient-based formulation is prefered to circumvent some limitations.

The proposed developments demonstrate that for slightly to moderately coupled nonlinear systems in a suitable modal basis, the equivalent linearized response can be seen as a convergent series of correction terms initiated with the stochastic response of a main decoupled linear system. This work shows that the concept of asymptotic expansion of a modal transfer matrix might be efficiently used to enhance the GEL technique. Indeed, this expansion allows to compute rapidly the Jacobian matrix required in a gradient-based method. The conditioning of the system is also improved, especially for large structures. The computational effort is thus attractively reduced, while preserving the advantages of spectral analysis.

Because simulation techniques or alternative exact approaches would equally perform in small-size structures with simple loadings, a specific attention in the developments is dedicated to high-dimensional structures subject to coherent random excitation fields such as those encountered in wind and earthquake engineering. The proposed method is capable of dealing with nonlinear conservative as well as dissipative forces, either affecting some degrees of freedom only, or more regularly distributed in the whole structure.

First, the philosophy of the equivalent linearization is exposed, then the asymptotic expansion of a modal transfer matrix is developed in the context of GEL. A Newton-Raphson procedure applying the asymptotic expansion is then described. Finally, illustrated examples are proposed to emphasize the pertinence of the method and to highlight the underlying assumptions. 


\section{Spectral strategy for stochastic linearization of large structures}

On a probability space $(\Theta, \mathfrak{F}, \mathbb{P})$, the equation of motion of an $n$-DOF nonlinear system is

$$
\mathbf{M} \ddot{\mathbf{y}}+\mathbf{C} \dot{\mathbf{y}}+\mathbf{K y}+\mathbf{g}(\mathbf{y}, \dot{\mathbf{y}})=\mathbf{f},
$$

where $\mathbf{M}, \mathbf{C}$ and $\mathbf{K}$ are the $n \times n$-dimensional mass, damping and stiffness matrices of the structure, respectively, $\mathbf{f}(t, \theta): \mathbb{R}^{+} \times \Theta \mapsto \mathbb{R}^{n}$ is the vector of the random exogenous Gaussian forces and the dot denotes the time derivative. The vector $\mathbf{y}(t, \theta): \mathbb{R}^{+} \times \Theta \mapsto \mathbb{R}^{n}$ gathers the nodal displacements expected to be non-Gaussian processes due to the nonlinear forces gathered in the vector function $\mathbf{g}(\mathbf{y}, \dot{\mathbf{y}}): \mathbb{R}^{n} \times \mathbb{R}^{n} \mapsto \mathbb{R}^{n}$. With this formalism, the equation of motion is split into four contributions : inertial forces, internal linear forces, internal nonlinear forces and exogenous random forces. Actually, in these developments, we consider nonlinear conservative or dissipative forces only depending on the nodal displacements of the structure (no history variable). Discarding the nonlinear forces $\mathbf{g}(\mathbf{y}, \dot{\mathbf{y}})$ in (1) produces a linear governing equation, refered to as the linear subsystem in the sequel.

In the considered problem, the size $n$ of the system is possibly large, and the exogeneous forces are characterized by a PSD matrix $\mathbf{S}_{\mathbf{f}}(\omega)$ with possibly complex expressions as typically encountered in realistic wind turbulence model [36] or spatial coherence in seismic engineering [37]. For the sake of clarity in the following analytical developments, only antisymmetric nonlinear forces and zero-mean excitation processes are considered. Otherwise, some minor modifications to the method must be operated to take into account the mean response and the noncentered statistical moments [10.

The stochastic linearization aims at replacing Equation (1) by the equation of motion of a $n$-DOF equivalent linear structure. The equivalent equation of motion reads

$$
\mathbf{M} \ddot{\mathbf{x}}+\left(\mathbf{C}+\mathbf{C}_{e q}\right) \dot{\mathbf{x}}+\left(\mathbf{K}+\mathbf{K}_{e q}\right) \mathbf{x}=\mathbf{f},
$$

where $\mathbf{x}$ and $\dot{\mathbf{x}}$ are the Gaussian nodal displacements and velocities of the equivalent linear system, respectively, and with $\mathbf{K}_{e q}$ and $\mathbf{C}_{e q}$ the equivalent stiffness and damping matrices, respectively [10. The probabilistic response of the system is thus completely characterized by the symmetric covariance matrices $\boldsymbol{\Sigma}_{\mathbf{x}}$ and $\boldsymbol{\Sigma}_{\dot{\mathbf{x}}}$, obtained by integration of the corresponding PSD matrices

$$
\boldsymbol{\Sigma}_{\mathbf{x}}=\int_{\mathbb{R}} \mathbf{S}_{\mathbf{x}} d \omega, \quad \text { and } \quad \Sigma_{\dot{\mathbf{x}}}=\int_{\mathbb{R}} \mathbf{S}_{\dot{\mathbf{x}}} d \omega
$$

which are themselves obtained by left- and right-multiplication of $\mathbf{S}_{\mathbf{f}}(\omega)$ by the nodal frequency response function of the system [38].

The equivalent stiffness and damping matrices in $(2)$ are determined by minimizing the error function $\mathcal{E}[8$, defined as

$$
\mathcal{E}=E\left[\left(\mathbf{K}_{e q} \mathbf{x}+\mathbf{C}_{e q} \dot{\mathbf{x}}-\mathbf{g}(\mathbf{x}, \dot{\mathbf{x}})\right)\left(\mathbf{K}_{e q} \mathbf{x}+\mathbf{C}_{e q} \dot{\mathbf{x}}-\mathbf{g}(\mathbf{x}, \dot{\mathbf{x}})\right)^{T}\right]
$$

with $E[$.$] the expectation operator. Because the covariance between displacements and velocities is equal to zero in$ a stationary setting $E\left[\mathbf{x} \dot{\mathbf{x}}^{T}\right]=\mathbf{0}$, the equivalent matrices $\mathbf{K}_{e q}$ and $\mathbf{C}_{e q}$ are respectively given by

$$
\mathbf{K}_{e q}=\boldsymbol{\Sigma}_{\mathbf{x}}^{-1} E\left[\mathbf{x g}(\mathbf{x}, \dot{\mathbf{x}})^{T}\right], \quad \text { and } \quad \mathbf{C}_{e q}=\boldsymbol{\Sigma}_{\dot{\mathbf{x}}}^{-1} E\left[\dot{\mathbf{x}} \mathbf{g}(\mathbf{x}, \dot{\mathbf{x}})^{T}\right] .
$$

For usual nonlinear mappings $\mathbf{g}$, provided they are explicitely known, the expectations in the above equations may be expressed as a function of $\boldsymbol{\Sigma}_{\mathbf{x}}$ and $\boldsymbol{\Sigma}_{\dot{\mathbf{x}}}$, in which case (5) also reads

$$
\mathbf{K}_{e q}=\mathcal{K}\left(\boldsymbol{\Sigma}_{\mathbf{x}}, \boldsymbol{\Sigma}_{\dot{\mathbf{x}}}\right), \quad \text { and } \quad \mathbf{C}_{e q}=\mathcal{C}\left(\boldsymbol{\Sigma}_{\mathbf{x}}, \boldsymbol{\Sigma}_{\dot{\mathbf{x}}}\right)
$$

with $\mathcal{K}$ and $\mathcal{C}$ two operators depending on the kind of nonlinearity in $\mathbf{g}$.

Equations (3) and (6) constitute a set of nonlinear algebraic equations that need to be solved for $\boldsymbol{\Sigma}_{\mathbf{x}}$ and $\boldsymbol{\Sigma}_{\dot{\mathbf{x}}}$. A classical iterative approach to the solution of that set ([10, p. 140) consists in initiating the unknown matrices $\mathbf{K}_{e q}$ and $\mathbf{C}_{e q}$ to zero, in which case the linear subsystem is analyzed, and using the fixed-point iterative scheme. In 
large scale problems, this traditional algorithm is readily implemented, but it requires the costly construction and integration of full matrices at each iteration step.

The response of a given linear structure can be computed using a limited number of $m$ normal modes of vibration, with $m \ll n$ for large scale structures. The costly construction (and then integration) of $\mathbf{S}_{\mathbf{x}}$ and $\mathbf{S}_{\dot{\mathbf{x}}}$ is thus replaced by

$$
\mathbf{S}_{\mathbf{x}}=\boldsymbol{\Phi} \mathbf{S}_{\mathbf{q}} \boldsymbol{\Phi}^{T}, \quad \text { and } \quad \mathbf{S}_{\dot{\mathbf{x}}}=\omega^{2} \boldsymbol{\Phi} \mathbf{S}_{\mathbf{q}} \boldsymbol{\Phi}^{T},
$$

where $\boldsymbol{\Phi}$ is the $n \times m$ matrix collecting the normal modes and $\mathbf{S}_{\mathbf{q}}(\omega)$ represents the psd matrix of modal coordinates. In principle, the normal modes should result from the eigenproblem $\left(\mathbf{K}+\mathbf{K}_{e q}\right) \boldsymbol{\Phi}=\omega^{2} \mathbf{M} \boldsymbol{\Phi}$, in which case they should be updated at each iteration, as $\mathbf{K}_{e q}$ changes. The possible saving on the construction and multiplication of large matrices is thus shifted to repeated eigenvalue decompositions. To use the modal basis related to matrices $\mathbf{K}+\mathbf{K}_{e q}$ and $\mathbf{M}$ is a natural reaction from linear dynamics aiming at uncoupling the resulting set of equations, on top of reducing the size of the problem. Since it is computationally expensive, this option is abandoned.

Instead, inspired by a similar approach within environments with moderate and non-essential nonlinear forces, it is suggested to work in a constant modal basis (as in [39]) resulting from the eigenproblem

$$
(\mathbf{K}+\tilde{\mathbf{K}}) \boldsymbol{\Phi}=\omega^{2} \mathbf{M} \boldsymbol{\Phi}
$$

where $\tilde{\mathbf{K}}$ is a fixed estimation of $\mathbf{K}_{e q}$ that accounts for the distribution of the nonlinear stiffness forces within the structure, in an equivalent manner, even if their intensity is a priori unknown. The construction of this matrix is discussed later in the light of relevant examples. This constant modal basis is normalized to have unit generalized masses, such that

$$
\boldsymbol{\Phi}^{T} \mathbf{M} \boldsymbol{\Phi}=\mathbf{I}, \quad \boldsymbol{\Phi}^{T} \mathbf{K} \boldsymbol{\Phi}=\mathbf{\Omega}
$$

with $\mathbf{I}$ the $m \times m$-identity matrix and $\boldsymbol{\Omega}$ a non-diagonal generalized stiffness matrix.

Using the modal superposition principle [38, Equation (2) reads

$$
\ddot{\mathbf{q}}+\left(\mathbf{D}+\mathbf{D}_{e q}\right) \dot{\mathbf{q}}+\left(\boldsymbol{\Omega}+\boldsymbol{\Omega}_{e q}\right) \mathbf{q}=\mathbf{p}
$$

where $\mathbf{q}(t)$ is the vector of modal coordinates $(\mathbf{x}=\mathbf{\Phi} \mathbf{q})$ and $\mathbf{D}=\boldsymbol{\Phi}^{T} \mathbf{C} \boldsymbol{\Phi}, \boldsymbol{\Omega}_{e q}=\boldsymbol{\Phi}^{T} \mathbf{K}_{e q} \boldsymbol{\Phi}, \mathbf{D}_{e q}=\boldsymbol{\Phi}^{T} \mathbf{C}_{e q} \boldsymbol{\Phi}$ and $\mathbf{p}(t)=\boldsymbol{\Phi}^{T} \mathbf{f}(t)$. Even though the number of variables has been reduced by the modal projection, the equations of motion are not decoupled because the matrices in $(10)$ are not necessarily diagonal matrices, even $\boldsymbol{\Omega}$, quite the opposite actually. Therefore, the uncoupling advantage offered by the modal projection is lost but this issue may be treated with existing solutions [26, as developed next.

The PSD matrix of modal coordinates $\mathbf{S}_{\mathbf{q}}(\omega)$ introduced in $(7)$ is given by

$$
\mathbf{S}_{\mathbf{q}}=\mathbf{H S}_{\mathbf{p}} \mathbf{H}^{*}
$$

where the superscript ${ }^{*}$ denotes the Hermitian operator and where the modal transfer matrix of the equivalent linearized system $\mathbf{H}(\omega): \mathbb{R} \mapsto \mathbb{C}^{m \times m}$ is defined as

$$
\mathbf{H}=\left(-\omega^{2} \mathbf{I}+\iota \omega\left(\mathbf{D}+\mathbf{D}_{e q}\right)+\boldsymbol{\Omega}+\boldsymbol{\Omega}_{e q}\right)^{-1}
$$

with $\iota=\sqrt{-1}$. The PSD matrix of modal loadings is obtained as $\mathbf{S}_{\mathbf{p}}=\boldsymbol{\Phi}^{T} \mathbf{S}_{\mathbf{f}} \boldsymbol{\Phi}$. The projection of the highdimensional random excitation field $\mathbf{f}(t)$ in the modal basis consitutes the major computational cost (i.e. $\mathcal{O}\left(n^{2}\right)$ ), for large structures as encountered in civil engineering. The projection of this field in a fixed and energy-independent basis is another key point in our formulation, that makes it rational for large structures. The modal loading $\mathbf{S}_{\mathbf{p}}(\omega)$ is indeed established once and for all, instead of being updated too, at each iteration.

With this modal approach, the set of nonlinear equations (6) is not supplemented with the heavy estimation of the covariance matrices $\boldsymbol{\Sigma}_{\mathbf{x}}$ and $\boldsymbol{\Sigma}_{\dot{\mathbf{x}}}$ as given in (3), but rather

$$
\boldsymbol{\Sigma}_{\mathbf{x}}=\boldsymbol{\Phi} \boldsymbol{\Sigma}_{\mathbf{q}} \boldsymbol{\Phi}^{T} ; \quad \boldsymbol{\Sigma}_{\dot{\mathbf{x}}}=\boldsymbol{\Phi} \boldsymbol{\Sigma}_{\dot{\mathbf{q}}} \boldsymbol{\Phi}^{T}
$$


where the covariance matrices of the modal coordinates as well as their time derivatives are

$$
\boldsymbol{\Sigma}_{\mathbf{q}}=\int_{\mathbb{R}} \mathbf{S}_{\mathbf{q}} d \omega ; \quad \boldsymbol{\Sigma}_{\dot{\mathbf{q}}}=\int_{\mathbb{R}} \omega^{2} \mathbf{S}_{\mathbf{q}} d \omega .
$$

The covariance matrices $\boldsymbol{\Sigma}_{\mathbf{x}}$ and $\boldsymbol{\Sigma}_{\dot{\mathbf{x}}}$ are still functions of the a priori unknown equivalent matrices $\mathbf{K}_{e q}$ and $\mathbf{C}_{e q}$ through $\mathbf{D}_{e q}$ and $\boldsymbol{\Omega}_{e q}$ in $(12)$ and the same fixed-point procedure may still be used to readily solve the set of equations (6)-(13). Nevertheless, as the convergence of the fixed-point algorithm is sensitive to the Jacobian of operators $\mathcal{K}$ and $\mathcal{C}$, with known evidences of slow convergence [35, the use of any gradient-based method is promoted which, at the expanse of heavier computational costs, offers a faster convergence and an increase of the convergence domain. This is formalized by transforming (14) into the canonical form

$$
\Sigma_{\mathbf{q}}=\mathcal{F}_{1}\left(\boldsymbol{\Sigma}_{\mathbf{q}}, \boldsymbol{\Sigma}_{\dot{\mathbf{q}}}\right) \quad ; \quad \boldsymbol{\Sigma}_{\dot{\mathbf{q}}}=\mathcal{F}_{2}\left(\boldsymbol{\Sigma}_{\mathbf{q}}, \boldsymbol{\Sigma}_{\dot{\mathbf{q}}}\right)
$$

As a difference with this alternative formulation, the unknowns of the problem are the elements of the modal covariance matrices. The operators $\mathcal{F}_{1}$ and $\mathcal{F}_{2}$ correspond to the integral operation in $(14)$, while $\mathbf{H}\left(\omega ; \boldsymbol{\Omega}_{e q}, \mathbf{D}_{e q}\right)$ is an implicit function of the unknown modal covariance matrices. A main objective of this paper is to provide an efficient formulation for the solution of 15 .

\section{Asymptotic expansion method with the modal spectral analysis}

\subsection{Derivation of the asymptotic formulation}

The governing equations in the modal basis $(10)$ are slightly to moderately coupled, depending on the intensity of nonlinear forces in the global balance of forces, as a result of the projection into a fixed basis. Former works have demonstrated the advantage of considering this kind of coupled set of modal equations as a perturbation of a main decoupled problem [25, 26]. In order to decouple the modal equation of motion of the equivalent linear structure, the modal damping and stiffness matrices are respectively split into two contributions such that

$$
\mathbf{D}+\mathbf{D}_{e q}=\mathbf{D}_{d}+\mathbf{D}_{o}
$$

and

$$
\boldsymbol{\Omega}+\boldsymbol{\Omega}_{e q}=\boldsymbol{\Omega}_{d}+\boldsymbol{\Omega}_{o},
$$

where $\mathbf{D}_{d}$ and $\mathbf{D}_{o}$ are built as $\mathbf{D}_{d}=\left(\mathbf{D}+\mathbf{D}_{e q}\right) \circ \mathbf{I}$ (diagonal elements) and $\mathbf{D}_{o}=\mathbf{D}+\mathbf{D}_{e q}-\mathbf{D}_{d}$ (out-of-diagonal elements) and where $\boldsymbol{\Omega}_{d}$ and $\boldsymbol{\Omega}_{o}$ are built as $\boldsymbol{\Omega}_{d}=\left(\boldsymbol{\Omega}+\boldsymbol{\Omega}_{e q}\right) \circ \mathbf{I}$ (diagonal elements) and $\boldsymbol{\Omega}_{o}=\boldsymbol{\Omega}+\boldsymbol{\Omega}_{e q}-\boldsymbol{\Omega}_{d}$ (out-of-diagonal elements), with $\circ$ the Hadamard product. With these notations, the modal transfer matrix (12) becomes

$$
\mathbf{H}=\left(\mathbf{J}_{d}+\mathbf{J}_{o}\right)^{-1},
$$

where

$$
\mathbf{J}_{d}=\boldsymbol{\Omega}_{d}-\omega^{2} \mathbf{I}+\iota \omega \mathbf{D}_{d}, \quad \text { and } \quad \mathbf{J}_{o}=\boldsymbol{\Omega}_{o}+\iota \omega \mathbf{D}_{o}
$$

gather the diagonal and out-of-diagonal elements, respectively. The elements of matrices $\mathbf{J}_{d}(\omega)$ and $\mathbf{J}_{o}(\omega)$ come from both the linear subsystem and the equivalent terms. The subscript $d$ recalls that these matrices are diagonal by construction.

The diagonal modal transfer matrix of the virtually decoupled system is also introduced as $\mathbf{H}_{d}(\omega)=\mathbf{J}_{d}^{-1}$. It corresponds to the modal transfer matrix that would be obtained if the coupling resulting from the projection in the generalized basis was simply neglected.

Substitution of (18) into (11) readily leads to

$$
\mathbf{S}_{\mathbf{q}}=\left(\mathbf{I}+\mathbf{H}_{d} \mathbf{J}_{o}\right)^{-1} \mathbf{S}_{\mathbf{q}_{d}}\left(\mathbf{I}+\mathbf{J}_{o}^{*} \mathbf{H}_{d}^{*}\right)^{-1}
$$

The virtual response of the decoupled system $\mathbf{S}_{\mathbf{q}_{d}}$, as would be obtained by only retaining diagonal elements in the modal matrices, is 


$$
\mathbf{S}_{\mathbf{q}_{d}}=\mathbf{H}_{d} \mathbf{S}_{\mathbf{p}} \mathbf{H}_{d}^{*} .
$$

Upon some smallness conditions on the product $\mathbf{H}_{d} \mathbf{J}_{o}$ formally studied in the following, Equation (18) may be written as

$$
\mathbf{H}=\mathbf{I}+\sum_{j=1}^{\infty}\left(-\mathbf{H}_{d} \mathbf{J}_{o}\right)^{j}
$$

We denote by asymptotic expansion-based method, all the developments based on 22] 25, 26]. Equation 20] now reads

$$
\mathbf{S}_{\mathbf{q}}=\left(\mathbf{I}+\sum_{k=1}^{\infty}\left(-\mathbf{H}_{d} \mathbf{J}_{o}\right)^{k}\right) \mathbf{S}_{\mathbf{q}_{d}}\left(\mathbf{I}+\sum_{j=1}^{\infty}\left(-\mathbf{J}_{o}^{*} \mathbf{H}_{d}^{*}\right)^{j}\right),
$$

transforming thus the inverse of full matrices into convergent series. This product of series is written as a single series which is truncated, for practical needs, at an order $N$ such that

$$
\mathbf{S}_{\mathbf{q}, N}=\mathbf{S}_{\mathbf{q}_{d}}+\sum_{k=1}^{N} \Delta \mathbf{S}_{\mathbf{q}, k}
$$

with $\Delta \mathbf{S}_{\mathbf{q}, k}$ the $k$-th correction term added to the decoupled approximation $\mathbf{S}_{\mathbf{q}_{d}}$. Thence, provided a given measure of $\mathbf{H}_{d} \mathbf{J}_{o}$ is of order $\epsilon$ (a small parameter), the same measure of the correction term $\Delta \mathbf{S}_{\mathbf{q}, k}$ is of order $\epsilon^{k}$. Working out the algebra yields

$$
\Delta \mathbf{S}_{\mathbf{q}, 1}(\omega)=-\mathbf{H}_{d} \mathbf{J}_{o} \mathbf{S}_{\mathbf{q}_{d}}-\mathbf{S}_{\mathbf{q}_{d}} \mathbf{J}_{o}^{*} \mathbf{H}_{d}^{*}
$$

for the first correction term and a recurrence relation

$$
\Delta \mathbf{S}_{\mathbf{q}, k}(\omega)=-\left(\mathbf{H}_{d} \mathbf{J}_{o} \Delta \mathbf{S}_{\mathbf{q}, k-1}+\Delta \mathbf{S}_{\mathbf{q}, k-1} \mathbf{J}_{o}^{*} \mathbf{H}_{d}^{*}\right)-\mathbf{H}_{d} \mathbf{J}_{o} \Delta \mathbf{S}_{\mathbf{q}, k-2} \mathbf{J}_{o}^{*} \mathbf{H}_{d}^{*}
$$

for $k \geq 2$ and $\Delta \mathbf{S}_{\mathbf{q}, 0}=\mathbf{S}_{\mathbf{q}_{d}}$. The correction terms do not require the inversion of any full matrix, because they are expressed in terms of the decoupled system.

Substitution of 24)-26 into (14) provides the asymptotic expansion of the covariance matrices $\boldsymbol{\Sigma}_{\mathbf{q}}$ and $\boldsymbol{\Sigma}_{\mathbf{q}}$ that read

$$
\boldsymbol{\Sigma}_{\mathbf{q}, N}=\boldsymbol{\Sigma}_{\mathbf{q}_{d}}+\sum_{k=1}^{N} \Delta \boldsymbol{\Sigma}_{\mathbf{q}, k} \quad ; \quad \boldsymbol{\Sigma}_{\dot{\mathbf{q}}, N}=\boldsymbol{\Sigma}_{\dot{\mathbf{q}}_{d}}+\sum_{k=1}^{N} \Delta \boldsymbol{\Sigma}_{\dot{\mathbf{q}}, k} .
$$

The first correction terms for the modal covariance matrices defined in Equation (14) read

$$
\Delta \boldsymbol{\Sigma}_{\mathbf{q}, 1}=-\left(\mathbf{L}_{\mathbf{q}, 1}+\mathbf{L}_{\mathbf{q}, 1}^{*}\right), \quad \Delta \boldsymbol{\Sigma}_{\dot{\mathbf{q}}, 1}=-\left(\mathbf{L}_{\dot{\mathbf{q}}, 1}+\mathbf{L}_{\dot{\mathbf{q}}, 1}^{*}\right)
$$

with

$$
\mathbf{L}_{\mathbf{q}, 1}=\int_{\mathbb{R}} \mathbf{H}_{d} \mathbf{J}_{o} \mathbf{S}_{\mathbf{q}_{d}} d \omega, \quad \mathbf{L}_{\dot{\mathbf{q}}, 1}=\int_{\mathbb{R}} \omega^{2} \mathbf{H}_{d} \mathbf{J}_{o} \mathbf{S}_{\mathbf{q}_{d}} d \omega
$$

Replacing the expression for $\mathbf{J}_{o}$ from $\left[19\right.$, the matrices $\mathbf{L}_{\mathbf{q}, 1}$ and $\mathbf{L}_{\mathbf{q}, 1}$ are finally expressed as

$$
\begin{gathered}
\left(\mathbf{L}_{\mathbf{q}, 1}\right)_{i j}=\sum_{k=1}^{m}\left(\boldsymbol{\Omega}_{o}\right)_{i k} \mathcal{I}_{i j k, 0}+\sum_{k=1}^{m}\left(\mathbf{D}_{o}\right)_{i k} \mathcal{I}_{i j k, 1} \\
\left(\mathbf{L}_{\dot{\mathbf{q}}, 1}\right)_{i j}=-\left(\sum_{k=1}^{m}\left(\boldsymbol{\Omega}_{o}\right)_{i k} \mathcal{I}_{i j k, 2}+\sum_{k=1}^{m}\left(\mathbf{D}_{o}\right)_{i k} \mathcal{I}_{i j k, 3}\right)
\end{gathered}
$$


with

$$
\mathcal{I}_{i j k, \alpha}=\int_{\mathbb{R}}(\iota \omega)^{\alpha}\left(\mathbf{H}_{d}\right)_{i}\left(\mathbf{H}_{d}\right)_{k}\left(\mathbf{S}_{\mathbf{p}}\right)_{k j}\left(\mathbf{H}_{d}^{*}\right)_{j} d \omega
$$

with $\alpha=0, \ldots 3$ and $i, j, k=1, \ldots m$. As a noteworthy advantage of this formulation, the integral $\mathcal{I}_{i j k, \alpha}$ only depends on the decoupled system and the out-of-diagonal elements of $\mathbf{J}_{o}$ are seen as higher-order perturbation sources of the decoupled system. The reason why the derivation ends up with such a simple formulation is that the whole concept is based on linear algebra. Retrospectively, the matrix inversions in 20 are replaced by linear combinations, thanks to the asymptotic expansion, and any operation in the derivation is linear with respect to its arguments. The permutation of these linear operators precisely allows the expression of the response of the coupled systems as a perturbation of the response of the main decoupled system. From an algorithmic point of view, the integrals $\mathcal{I}_{i j k, \alpha}$ are promptly established at each iteration. As explained next, they are also stored in order to set up the Jacobian matrix in the iterative solution of the problem.

Similarly, the second correction terms for the modal covariance matrices read

$$
\Delta \boldsymbol{\Sigma}_{\mathbf{q}, 2}=\left(\mathbf{L}_{\mathbf{q}, 2, I}+\mathbf{L}_{\mathbf{q}, 2, I}^{*}+\mathbf{L}_{\mathbf{q}, 2, I I}\right), \quad \Delta \boldsymbol{\Sigma}_{\dot{\mathbf{q}}, 2}=\left(\mathbf{L}_{\dot{\mathbf{q}}, 2, I}+\mathbf{L}_{\dot{\mathbf{q}}, 2, I}^{*}+\mathbf{L}_{\dot{\mathbf{q}}, 2, I I}\right)
$$

with

$$
\begin{array}{cc}
\mathbf{L}_{\mathbf{q}, 2, I}=\int_{\mathbb{R}}\left(\mathbf{H}_{d} \mathbf{J}_{o}\right)^{2} \mathbf{S}_{\mathbf{q}_{d}} d \omega, & \mathbf{L}_{\mathbf{q}, 2, I I}=\int_{\mathbb{R}} \mathbf{H}_{d} \mathbf{J}_{o} \mathbf{S}_{\mathbf{q}_{d}} \mathbf{J}_{o}^{*} \mathbf{H}_{d}^{*} d \omega \\
\mathbf{L}_{\dot{\mathbf{q}}, 2, I}=\int_{\mathbb{R}} \omega^{2}\left(\mathbf{H}_{d} \mathbf{J}_{o}\right)^{2} \mathbf{S}_{\mathbf{q}_{d}} d \omega, & \mathbf{L}_{\dot{\mathbf{q}}, 2, I I}=\int_{\mathbb{R}} \omega^{2} \mathbf{H}_{d} \mathbf{J}_{o} \mathbf{S}_{\mathbf{q}_{d}} \mathbf{J}_{o}^{*} \mathbf{H}_{d}^{*} d \omega .
\end{array}
$$

The computation of the second correction term is recommended but not mandatory for all terms. Usually the first correction is sufficient for the estimation of out-of-diagonal terms in $\Delta \boldsymbol{\Sigma}_{\mathbf{q}}$ and $\Delta \boldsymbol{\Sigma}_{\mathbf{q}}$ whilst the second correction offers a better estimation of diagonal elements (variances). This general trend should be regarded with a discussion on the coherence in the generalized loading [26].

\subsection{Discussion on the convergence of the asymptotic expansion}

The conditions upon which the series expansion derived above shall be valid are now reviewed. A necessary and sufficient condition for the series expansions in 22 to converge is that

$$
\Gamma\left[\mathbf{H}_{d}(\omega) \mathbf{J}_{o}(\omega)\right]<1, \quad \forall \omega \in \mathbb{R}
$$

with $\Gamma[\mathbf{A}]=\max \left\{\left|\lambda_{\mathbf{A}, i}\right|, i=1, \ldots m\right\}$ the spectral radius of $\mathbf{A} \in \mathbb{C}^{m \times m}$ and $\lambda_{\mathbf{A}, i}$ the $i$-th eigenvalue of $\mathbf{A}$ [0]. The spectral radius of $\mathbf{H}_{d} \mathbf{J}_{o}$ also defines the index of diagonality $\rho$ of $\mathbf{J}_{d}+\mathbf{J}_{o}$ [40, 41]

$$
\Gamma\left[\mathbf{H}_{d} \mathbf{J}_{o}\right]=\rho\left[\mathbf{J}_{d}+\mathbf{J}_{o}\right],
$$

as it measures the relative importance of the diagonal terms in $\mathbf{J}_{d}$ compared with the out-of-diagonal ones in $\mathbf{J}_{o}$.

The general condition formulated in $(36)$ must be checked for all the values of $\omega \in \mathbb{R}$. It is relevant to first investigate the limit behaviors for $\omega$ tending to zero and to infinity, such that

$$
\begin{gathered}
\lim _{\omega \rightarrow 0} \mathbf{H}_{d} \mathbf{J}_{o}=\lim _{\omega \rightarrow 0}\left(\boldsymbol{\Omega}_{d}-\omega^{2} \mathbf{I}+\iota \omega \mathbf{D}_{d}\right)^{-1} \mathbf{J}_{o}=\boldsymbol{\Omega}_{d}^{-1} \boldsymbol{\Omega}_{o} \\
\lim _{|\omega| \rightarrow+\infty} \mathbf{H}_{d} \mathbf{J}_{o}=\lim _{\omega \rightarrow+\infty}-\frac{1}{\omega^{2}} \mathbf{J}_{o}=\mathbf{0} .
\end{gathered}
$$

These conditions are related to the quasi-static and the inertial regimes of the linear system, respectively. The smallness of the product $\mathbf{H}_{d} \mathbf{J}_{o}$ is ensured for high-frequency responses, while the smallness of $\boldsymbol{\Omega}_{d}^{-1} \boldsymbol{\Omega}_{o}$ is required for the quasi-static response. In this case, the spectral radius of $\boldsymbol{\Omega}_{d}^{-1} \boldsymbol{\Omega}_{o}$ is also the diagonality index of $\boldsymbol{\Omega}+\boldsymbol{\Omega}_{e q}$. If the spectral radius of $\boldsymbol{\Omega}_{d}^{-1} \boldsymbol{\Omega}_{o}$ is less than one, 


$$
\rho\left[\boldsymbol{\Omega}+\boldsymbol{\Omega}_{e q}\right]=\Gamma\left[\boldsymbol{\Omega}_{d}^{-1} \boldsymbol{\Omega}_{o}\right]<1,
$$

the matrix $\boldsymbol{\Omega}+\boldsymbol{\Omega}_{e q}$ is said to be diagonal dominant [40]. However, the criterion (40) is only sufficient for $\omega \rightarrow 0$.

Concerning the resonant regime occuring for $\omega$ equal to a natural frequency of the decoupled system, a straight criterion is not easily derived. And yet, the Gerschgorin theorem [40] allows to show that (i) all the Gerschgorin discs are concentric and centered on zero regardless of $\omega$ and that (ii) the largest disc is one of the discs calculated for $\omega$ equal to a natural frequency of the decoupled system. Therefore, the largest eigenvalue of $\mathbf{H}_{d} \mathbf{J}_{o}$ can be potentially found in one of these discs. The maximum value of $\Gamma\left[\mathbf{H}_{d} \mathbf{J}_{o}\right]$ is more judiciously sought in the biggest

disc, which is obtained for $\omega_{d, i}\left(=\left(\boldsymbol{\Omega}_{d}\right)_{i}^{1 / 2}\right)$. The convergence criterion $\rho_{\mathbf{J}}$ is a key indicator in the method. It must be calculated in each application to check the validity of the expansion, as done in the following. This explanation has the drawback to not properly justify the convergence criterion in terms of eigenvalues, but it is quite hard to calculate them explicitely, a reason for rather using a theorem allowing to bound them, at least.

The convergence of the series might be evaluated with the condition

$$
\rho_{\mathbf{J}}=\max \left\{\Gamma_{d, i}=\Gamma\left[\mathbf{H}_{d}\left(\omega_{d, i}\right) \mathbf{J}_{o}\left(\omega_{d, i}\right)\right], i=1, \ldots m\right\}<1 .
$$

After the numerical computation of the spectral radii $\Gamma_{d, i}$, the verification that they are local maxima can be easily performed (see [40]).

\section{Newton's iterative procedure and computational aspects}

In this Section, the application of Newton's algorithm to solve (15) is discussed, in the light of the asymptotic expansion formulation. As a major outcome, it will be observed that, again, the linear algebra, deliberately kept in the former derivation, results in a particularly efficient formulation.

\subsection{Asymptotic approximation of the Jacobian matrix}

Due to the symmetry of the modal covariance matrices $\boldsymbol{\Sigma}_{\mathbf{q}}$ and $\boldsymbol{\Sigma}_{\dot{\mathbf{q}}}, m(m+1)$ unknown elements are identified in the set of equations (15). For the sake of simplicity in the notations, they are gathered in the $m(m+1)$-vector of unknowns $\mathbf{u}$. As the matrix operators $\mathcal{F}_{1}$ and $\mathcal{F}_{2}$ are symmetric as well, they are similarly rearranged into a vector operator $\mathcal{F}$ as for the covariance matrices. Finally, the set of equations 15 is written in the residual form

$$
\mathcal{R}(\mathbf{u})=\mathbf{u}-\mathcal{F}(\mathbf{u})=\mathbf{0} .
$$

The operator $\mathcal{F}$ is nonlinear and involves the integrals defined in (14). Newton's algorithm to solve (42) requires the computation of a Jacobian matrix $\mathbf{T}$, defined as

$$
\mathbf{T}(\mathbf{u})=\nabla_{\mathbf{u}} \mathcal{R}^{\mathrm{T}}=\mathbf{I}-\nabla_{\mathbf{u}} \mathcal{F}^{\mathrm{T}}
$$

Starting from an initial guess $\mathbf{u}^{(0)}$, which is the solution of the linear subsystem in this case, a new iterate $\mathbf{u}^{(k+1)}$ is obtained by

$$
\mathbf{u}^{(k+1)}=\mathbf{u}^{(k)}-\mathbf{T}\left(\mathbf{u}^{(k)}\right)^{-\mathrm{T}} \mathcal{R}\left(\mathbf{u}^{(k)}\right), \quad k \geq 0
$$

and the procedure is repeated until a certain norm of $\mathcal{R}(\mathbf{u})$ becomes smaller than a desired threshold.

The formal derivation of $\mathbf{T}$ in the general case is rather cumbersome. For instance, the construction of $\nabla_{\mathbf{u}} \mathcal{F}^{\mathrm{T}}$, in the upper half of the Jacobian matrix (i.e. related to $\mathcal{F}_{1}$ ), requires the estimation of

$$
\nabla_{\mathbf{u}}\left(\int_{\mathbb{R}} \mathbf{H S}_{\mathbf{p}} \mathbf{H}^{*} d \omega\right)=\int_{\mathbb{R}}\left(\nabla_{\mathbf{u}} \mathbf{H}\right) \mathbf{S}_{\mathbf{p}} \mathbf{H}^{*} d \omega+\int_{\mathbb{R}} \mathbf{H S}_{\mathbf{p}}\left(\nabla_{\mathbf{u}} \mathbf{H}^{*}\right) d \omega,
$$

where $\nabla_{\mathbf{u}} \mathbf{H}$ further requires the application of the chain rule differentiation, as $\mathbf{H}\left(\omega ; \boldsymbol{\Omega}_{e q}, \mathbf{D}_{e q}\right)$ defined with respect to $\boldsymbol{\Omega}_{e q}$ and $\mathbf{D}_{e q}$ is differentiated with respect to $\mathbf{u}$. As a compensation, a finite difference method may be used to estimate the Jacobian matrix $\mathbf{T}\left(\mathbf{u}^{(k)}\right)$. However, this finite difference operation constitutes a heavy computational 
step in the method, precisely because the function estimation itself is already heavy. Indeed, for each perturbed component of the vector $\mathbf{u}^{(k)}$, the almost full modal transfer matrix must be inverted for every circular frequency and then the integration of the psd matrices $\mathbf{S}_{\mathbf{q}}$ and $\mathbf{S}_{\mathbf{q}}$ must be performed. The repeated inversions and integrations make this method prima facie quite inefficient, a reason why the fixed-point method might be preferred.

Interestingly however, the previous asymptotic developments can be used to simplify the computation of the Jacobian matrix and to offer, with a much less expensive algorithm, the quality of a second-order method. According to the previous developments, the vector $\mathcal{F}(\mathbf{u})$ is replaced by its first-order asymptotic expansion in (42). In this case, the Jacobian matrix may be analytically and efficiently approximated. Indeed, the construction of $\nabla_{\mathbf{u}} \mathcal{F}^{\mathrm{T}}$ in (43), requires the estimation of $\nabla_{\mathbf{u}} \mathbf{H}$ which is now regarded as

$$
\nabla_{\mathbf{u}} \mathbf{H} \simeq \nabla_{\mathbf{u}}\left(\left(\mathbf{I}-\mathbf{H}_{d} \mathbf{J}_{o}\right) \mathbf{H}_{d}\right) \simeq-\mathbf{H}_{d}\left(\nabla_{\mathbf{u}} \boldsymbol{\Omega}_{e q}+\iota \omega \nabla_{\mathbf{u}} \mathbf{D}_{e q}\right) \mathbf{H}_{d}
$$

where the two approximations correspond to first-order developments. According to the approximation (46), Equation (45) may read

$$
\nabla_{\mathbf{u}}\left(\int_{\mathbb{R}} \mathbf{H S}_{\mathbf{p}} \mathbf{H}^{*} d \omega\right) \simeq\left(\nabla_{\mathbf{u}} \mathbf{L}_{\mathbf{q}}\right)+\left(\nabla_{\mathbf{u}} \mathbf{L}_{\mathbf{q}}^{*}\right)
$$

with

$$
\left(\nabla_{\mathbf{u}} \mathbf{L}_{\mathbf{q}}\right)_{i j}=\sum_{k=1}^{m}\left(\nabla_{\mathbf{u}} \boldsymbol{\Omega}_{e q}\right)_{i k} \mathcal{I}_{i j k, 0}+\sum_{k=1}^{m}\left(\nabla_{\mathbf{u}} \mathbf{D}_{e q}\right)_{i k} \mathcal{I}_{i j k, 1}
$$

and $\mathcal{I}_{i j k, \alpha}$ exactly the same as defined in (32). Similarly, the second half of the Jacobian matrix, which is related to operator $\mathcal{F}_{2}$, is filled with $\nabla_{\mathbf{u}} \mathbf{L}_{\dot{\mathbf{q}}}+\nabla_{\mathbf{u}} \mathbf{L}_{\mathbf{q}}^{*}$, where

$$
\left(\nabla_{\mathbf{u}} \mathbf{L}_{\dot{\mathbf{q}}}\right)_{i j}=\sum_{k=1}^{m}\left(\nabla_{\mathbf{u}} \boldsymbol{\Omega}_{e q}\right)_{i k} \mathcal{I}_{i j k, 2}+\sum_{k=1}^{m}\left(\nabla_{\mathbf{u}} \mathbf{D}_{e q}\right)_{i k} \mathcal{I}_{i j k, 3} .
$$

A final substitution into (43) with the corresponding matrix-to-vector rearrangement provides an approximation of the Jacobian matrix, which is an edgestone in this Newton algorithm. Indeed, the numerical construction of this matrix is efficiently performed, since (i) it mainly relies on the scalars $\mathcal{I}_{i j k, \alpha}$ which are previouslycomputed for the calculation of $\mathcal{R}\left(\mathbf{u}^{(k)}\right)$, (ii) no matrix is inverted and (iii) no additionnal integral is performed. Only the results computed for a given guess of $\mathbf{u}$ in the iterative procedure are exploited. The gradients $\nabla_{\mathbf{u}} \boldsymbol{\Omega}_{e q}$ and $\nabla_{\mathbf{u}} \mathbf{D}_{e q}$ can be analytically established or with a finite difference scheme. We shall notice that this approximation of the Jacobian matrix can be applied, even if the operator $\mathcal{F}$ is not asymptotically approximated.

All in all, the derivation of the Jacobian matrix in this Section shows that, with the projection of the motion into a fixed and suitable modal basis, together with the asymptotic expansion, it is possible to rapidly construct an accurate first-order approximation of the Jacobian matrix, allowing to conveniently use a gradient-based method. Compared to the finite difference estimation of the full residual vector, the proposed method efficiently concentrates the time-consuming and ill-conditionned operations (matrix inversion and integration) in analytical developments.

It is also remarkable that the proposed method does not require any user-tuned continuation procedure to converge towards the solution. In the problems investigated by the authors, among which some are reported in Section 5 , a part of the solution of the linear subsystem is always used as initial guess $\mathbf{u}^{(0)}$. While the fixed-point algorithm is only able to handle limited nonlinearities in amplitude, the proposed algorithm smoothly converges towards the solution of the problem, without recoursing to any relaxation nor backtracking artifact.

\subsection{Solver implementation}

In order to make as clear as possible the implementation of the Newton algorithm, we propose to summarize in five steps the different operations that must be applied to perform the equivalent linearization with the asymptotic expansion-based method. We shall notice that the generalized basis $\mathbf{\Phi}$ can be modified throughout the procedure, since the value of the equivalent stiffness forces is a priori unknown.

Step 0. To initialize the problem, we first solve the eigenvalue problem (8) related to the linear subsystem with $\tilde{\mathbf{K}}^{(0)}=\mathbf{0}$. We project the linear equation of motion in the basis $\boldsymbol{\Phi}^{(0)}$, as well as the Gaussian force vector $\mathbf{f}$. We 
perform the spectral analysis 11 with $\mathbf{D}_{e q}=\boldsymbol{\Omega}_{e q}=\mathbf{0}$ in 10 . Finally, we obtain the matrices $\boldsymbol{\Sigma}_{\mathbf{q}}^{(0)}$ and $\boldsymbol{\Sigma}_{\dot{\mathbf{q}}}^{(0)}$ gathered in $\mathbf{u}^{(0)}$.

Step 1. We consider the $k$-th step $(k \geq 0)$ of the iteration procedure and the $l$-th generalized basis $\boldsymbol{\Phi}^{(l)}$ $(k \geq l \geq 0)$. We make the distinction between $k$ and $l$, since the basis is not necessarily adapted at each iteration. The elements of the matrices $\boldsymbol{\Sigma}_{\mathbf{q}, N}^{(k)}$ and $\boldsymbol{\Sigma}_{\mathbf{\mathbf { q }}, N}^{(k)}$ (as well as $\boldsymbol{\Sigma}_{\mathbf{x}, N}^{(k)}$ and $\boldsymbol{\Sigma}_{\dot{\mathbf{x}}, N}^{(k)}$ ) are known and gathered in $\mathbf{u}^{(k)}$. The equivalent matrices $\mathbf{D}_{e q}^{(k)}$ and $\boldsymbol{\Omega}_{e q}^{(k)}$ are computed with 6 . We can thus identify the matrices $\mathbf{D}_{o}^{(k)}, \mathbf{D}_{d}^{(k)}, \boldsymbol{\Omega}_{o}^{(k)}$ and $\boldsymbol{\Omega}_{d}^{(k)}$ by invoking 16 and 17 . According to 41 , we check whether the convergence criterion is verified, or not. If $\rho_{\mathbf{J}}<1$, we go to Step 3. Otherwise, we go to Step 2 to adapt the modal basis.

Step 2. In order to ensure the convergence of the asymptotic expansion, the modal basis may be adapted. It essentially means that the equivalent stiffness forces cease to be negligible compared with the linear ones in the basis $\boldsymbol{\Phi}^{(l)}$. A new generalized basis $\boldsymbol{\Phi}^{(l+1)}$ can be computed according to 8 with $\tilde{\mathbf{K}}^{(l+1)}=\mathbf{K}_{e q}^{(k)}$. In this case, the covariance matrices and the vector $\mathbf{u}^{(k)}$ must be evaluated in the new modal basis. We may identify two possible methods : (i) a new spectral analysis is performed according to (11) with the asymptotic expansion-based method or (ii) a linear mapping can be used to express the covariance matrices in the new modal basis. In this latter case, the nodal displacements are expressed in the modal basis $\boldsymbol{\Phi}^{(l+1)}$. However, this mapping requires to compute the (pseudo-) inverse of the mode shape matrix. Then, we go to Step 3.

Step 3. At this step, we estimate the operator $\mathcal{F}\left(\mathbf{u}^{(k)}\right)$. We apply the asymptotic expansion-based method to the modal transfer matrix $\mathbf{H}\left(\omega ; \boldsymbol{\Omega}_{e q}^{(k)}, \mathbf{D}_{e q}^{(k)}\right)$. We compute the decoupled approximation and the correction terms. By the way, we also compute the integrals $\mathcal{I}_{i j k, \alpha}$. If a suitable norm of the residual function $\mathcal{R}\left(\mathbf{u}^{(k)}\right)$ is reached, we stop the iteration procedure. Otherwise, we go back to Step 4.

Step 4. We apply the Newton algorithm to find the new guess of $\mathbf{u}^{(k+1)}$. Since we know from Step 3 the integrals $\mathcal{I}_{i j k, \alpha}$, we can directly compute the first-order approximation of the Jacobian matrix $\mathbf{T}\left(\mathbf{u}^{(k)}\right)$. By applying Equation 44 , we obtain $\mathbf{u}^{(k+1)}$ and we go to Step 1 .

With this short description for an implementation of the method, we understand how important is the convergence criterion $\rho_{\mathbf{J}}$. Actually, the method depends on the ability to find a suitable and convenient modal basis to project and decouple the equations of motion. Therefore, the proposed method is more pertinently applied to moderate modal coupling coming from the structural nonlinear behaviors. This modal coupling depends on both the intensity of the equivalent forces and their spatial distribution within the structures. These two facts are both measured with the index $\rho_{\mathbf{J}}$.

\subsection{Integration of the correction terms}

The proposed formulation relies on the estimation of the definite integrals $\mathcal{I}_{i j k, \alpha}$ and, to a lesser extent $\mathbf{L}_{\mathbf{q}, 2}$ and $\mathbf{L}_{\dot{\mathbf{q}}, 2}$, as defined previously. Taking into account the symmetry of the integrand, integration can be carried out on $\mathbb{R}^{+}$with available numerical integration methods [42. The only subtlety in the estimation of these integrals is related to the peakiness of the integrand which requires an adaptive integration scheme [35]. Actually a nonadaptive scheme, with a proper selection of the integration points, could also be developed since the poles of the integrands are easily identifiable, another consequence of the decoupling procedure since the integrands are the mixed products of the transfer functions of single degree-of-freedom systems. Although this numerical procedure is the standard application of the proposed method, the estimation of these integrals may be simplified (e.g. in the case that many modes are kept in the analysis), as underlined hereinafter.

First, for some kind of excitations, Jordan's lemma and Cauchy's residue theorem [43] may provide manageable explicit expressions. For instance, if the excitations are uncorrelated white noises, the PSD matrix $\mathbf{S}_{\mathbf{p}}$ is full of frequency-independent elements. This idea is inspired by well-known integrals (e.g. in the the CQC combination in earthquake engineering 38), such as

$$
\int_{\mathbb{R}}\left(\mathbf{H}_{d}\right)_{i}\left(\mathbf{S}_{\mathbf{p}}\right)_{i j}\left(\mathbf{H}_{d}^{*}\right)_{j} d \omega=\left(\mathbf{S}_{\mathbf{p}}\right)_{i j} \frac{4 \pi\left(\xi_{i} \omega_{d, i}+\xi_{j} \omega_{d, j}\right)}{\left(\omega_{d, i}^{2}-\omega_{d, j}^{2}\right)^{2}+4 \omega_{d, i} \omega_{d, j}\left(\xi_{i} \omega_{d, i}+\xi_{j} \omega_{d, j}\right)\left(\xi_{j} \omega_{d, i}+\xi_{i} \omega_{d, j}\right)}
$$


with $\left(\boldsymbol{\Omega}_{d}\right)_{i}=\omega_{d, i}^{2}$ and $\left(\mathbf{D}_{d}\right)_{i}=2 \xi_{i} \omega_{d, i}$ and may be extended to the computation of the integrals $\mathcal{I}_{i j k, \alpha}$.

A second case concerns loadings acting on a timescale different from the structural response. Inspired by the background-resonant approximation introduced by Davenport [44 for single degree-of-freedom systems, the method has been extended to other classes of problems [45. The required assumption that different frequency bands contribute independently to the total definite integral, may also be applied to estimate the integrals $\mathcal{I}_{i j k, \alpha}$. For instance, the integrals $\mathcal{I}_{i i i, 0}$ and $\mathcal{I}_{i i j, 0}$ may be approximated by

$$
\begin{gathered}
\mathcal{I}_{i i i, 0} \approx \frac{\left(\boldsymbol{\Sigma}_{\mathbf{p}}\right)_{i i}}{\omega_{d, i}^{6}}+\frac{\pi}{4 \xi_{i} \omega_{d, i}^{5}}\left(\left(\mathbf{S}_{\mathbf{p}}\left(\omega_{d, i}\right)\right)_{i i}-\frac{\omega_{d, i}}{2}\left(\mathbf{S}_{\mathbf{p}}^{\prime}\left(\omega_{d, i}\right)\right)_{i i}\right) \\
\mathcal{I}_{i i j, 0} \approx \frac{\left(\boldsymbol{\Sigma}_{\mathbf{p}}\right)_{j i}}{\omega_{d, i}^{4} \omega_{d, j}^{2}}+\frac{\pi}{2 \xi_{i} \omega_{d, i}^{3}} \Re\left(\left(\mathbf{S}_{\mathbf{p}}\left(\omega_{d, i}\right)\right)_{j i}\left(\mathbf{H}_{d}\left(\omega_{d, i}\right)\right)_{j}\right) .
\end{gathered}
$$

They are thus replaced by direct function evaluations, which indeed offers a considerable computational speedup. A comprehensive statement of this method and the theoretical basements are not exposed in this paper, but some highlights and further results are given in AppendixB

\section{Illustrations}

Three examples are next considered to illustrate the accuracy of the asymptotic expansion-based approach for various natures and intensities of nonlinear forces. As the truncation order and the convergence of the asymptotic expansion remain central in the method, the accuracy and the efficiency of the proposed method (with a finite number $N$ of terms) are assessed by comparison with the formal statistical linearization based on a fully coupled modal approach $(N=\infty)$ in which case a classical fixed-point method is also applied. In the following, the error between the different approaches is expressed as the ratio of the Euclidean norm of the difference between the two approaches over the same norm of the formal statistical linearization (supposed to be exact). The numerical efficiency of the method and its robustness are also investigated.

Although it is deliberately kept as a side-discussion, for each example, a full nodal step-by-step solution using a nonlinear Newmark scheme is also performed in order to indicate the validity of the statistical linearization. In any case, the discrepancies between the two statistical linearization approaches (the proposed one, and the formal one) is one order of magnitude smaller than the discrepancy between the Monte Carlo simulation and the formal statistical linearization.

\subsection{Mitigation of the along-wind response of high-rise building}

Mitigation of the along-wind response of high-rise buildings can be achieved with different types of damping devices such as Tuned Liquid Column Dampers (TLCD) [46]. The nonlinear governing equation of the liquid elevation $z$ in the TLCD due to the horizontal displacement $x$ of the liquid column [47] is given by

$$
\rho A L \ddot{z}+\frac{1}{2} \rho A \delta|\dot{z}| \dot{z}+2 \rho A g z=-\rho A B \ddot{x}
$$

where $\rho, A, B, L$ are the density, cross-sectional area, width and length of the liquid column, respectively and $\delta$, $g$ are the head loss coefficient and acceleration of gravity, respectively. As experiments have demonstrated that nonlinearity at the orifice remains small [18, the equivalent linearization is efficiently used to design such devices. Applying the statistical linearization, the equivalent equation to 53 reads

$$
\rho A L \ddot{z}+\sqrt{\frac{2}{\pi}} \rho A \delta \sigma_{\dot{z}} \dot{z}+2 \rho A g z=-\rho A B \ddot{x} .
$$

For the illustration, a 370m-high TV tower is analyzed under turbulent wind actions. This structure is an example proposed in [18, where the characteristics of the structure and TLCD are exhaustively reported. The spectrum of longitudinal turbulence used in this illustration is the model proposed by Davenport [4], such that

$$
\left(\mathbf{S}_{\mathbf{f}}\right)_{i j}=\frac{12}{\pi} K_{0}\left(\frac{1}{2} C_{a} \rho_{a} V_{10}^{2}\right)^{2}\left(\frac{h_{i} h_{j}}{100}\right)^{\beta} A_{i} A_{j} \operatorname{coh}\left(h_{i}, h_{j}, \omega\right) S_{v}(\omega),
$$


where the coherence function and the unilateral wind PSD are defined as

$$
\operatorname{coh}\left(h_{i}, h_{j}, \omega\right)=\exp \left(-\frac{C_{1}|\omega|}{2 \pi} \frac{\left|h_{i}-h_{j}\right|}{V_{10}}\right), \quad S_{v}(\omega)=\frac{4 \pi \bar{t}^{2}}{3 \omega\left(1+\bar{t}^{2}\right)^{4 / 3}}
$$

respectively, with $\bar{t}=600 \omega / \pi V_{10}, V_{10}$ the reference mean wind velocity $(\mathrm{m} / \mathrm{s})$ at $10 \mathrm{~m}$ above the ground, $\rho_{a}=1.2$ the air density, $K_{o}=0.007$ the surface drag coefficient, $C_{a}=0.7$ the drag coefficient; the other constants are $C_{1}=7$ and $\beta=0.15$. The height $h_{i}$ above the ground of the $i$-th story is calculated assuming an individual story height of $4 \mathrm{~m}$. The wind area of each floor is equal to $A_{i}=192 \mathrm{~m}^{2}$ and the velocity $V_{10}$ to $26.4 \mathrm{~m} / \mathrm{s}$.

Because no nonlinear stiffness force is considered in this example, the modal basis is the one of the linear subsystem $(\tilde{\mathbf{K}}=\mathbf{0})$. Figure 1 (a) illustrates the standard deviations of the horizontal deflections of the tower if the head loss coefficient $\delta$ is set equal to zero or equal to $30 \%$ (tuned value). With the TLCD, the standard deviation of the top displacement is nearly divided by two, which indicates the importance of the nonlinear damping. Figure 1.(b) illustrates the relative errors made with the asymptotic approach $(N=1,2)$ in comparison with a full matrix inversion $(N=\infty)$. With only the first correction term, relative errors range in $[-2 \% ;-1 \%]$, while the addition of the second correction term reduces the relative errors to less than $-0.5 \%$.
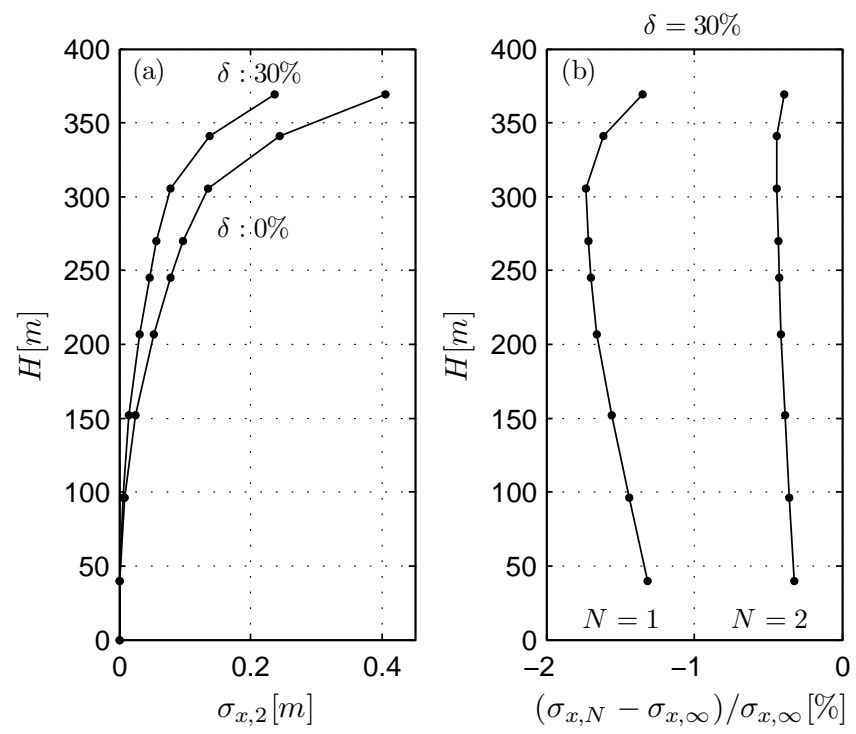

Figure 1: (a) Standard deviation of the horizontal displacements obtained with the equivalent linearization $(N=2)$ for $\delta=0 \%$ and $\delta=30 \%$. (b) Relative errors between the proposed asymptotic approach $(N=1,2)$ and the formal equivalent linearization $(N=\infty)$ for $\delta=30 \%$.

The correlation coefficient between the liquid elevation and the top displacement is shown in Fig. 2.(a). It increases rapidly with $\delta$, then tends to an asymptotic value. For this quantity, large relative errors, up to $8 \%$, occur with a first-order decoupling approximation, depicted in Fig. 2f(b). Once again, the first two correction terms are necessary to fit the reference values with relative errors lower than $-0.5 \%$.

Figure 3 shows the global increase of the damping ratios with the parameter $\delta$. The largest increase is observed for the first two modes, because the TLCD is tuned to the fundamental one. For the second mode, the damping is nearly multiplied by five for $\delta=30 \%$. The index of diagonality starts at 0.15 because the structural damping is not proportional and for $\delta=30 \%$ it increases up to 0.4 . 

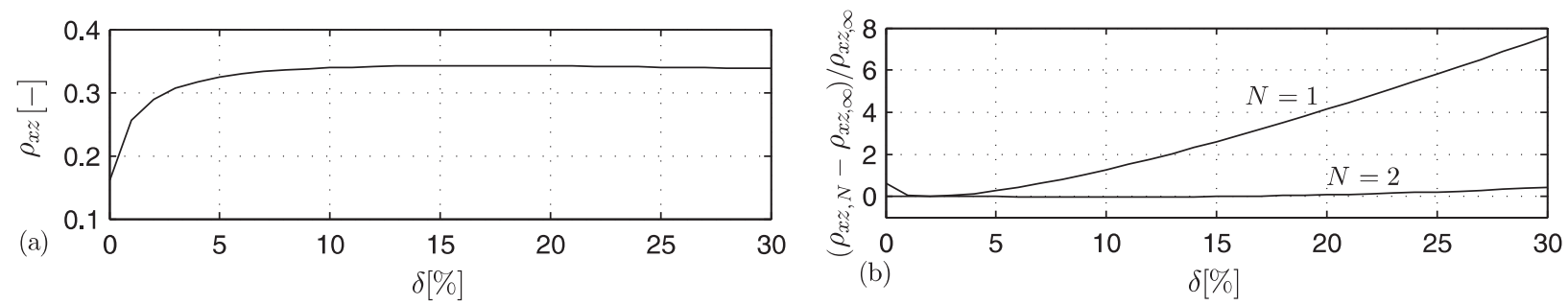

Figure 2: (a) Correlation coefficient between the liquid elevation $z$ of the liquid column and the top displacement $(N=2)$. (b) Relative errors [\%] between the decoupling asymptotic approach $(N=1,2)$ and the full matrix inversion $(N=\infty)$.
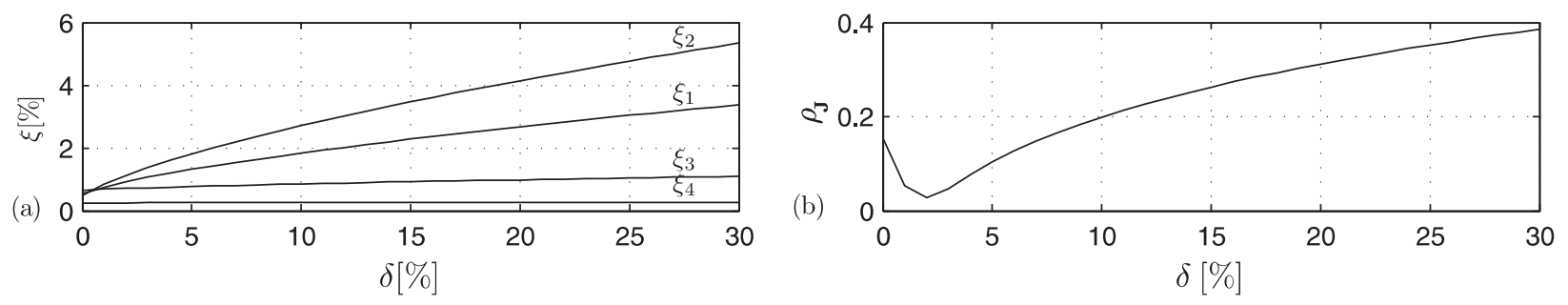

Figure 3: (a) Modal damping ratios and (b) index of diagonality as function of $\delta$.

The comparison with fully nonlinear time-domain simulations gives relative discrepancies lower than $4 \%$ in magnitude for the highest value of head loss coefficient $(\delta=30 \%)$. Actually, the nonlinear behaviour due to the orifices is weak and the global response of the structure is nearly Gaussian. In any case, the proposed method is closer to the solution of the equivalent linearization, than this latter one is from the Monte Carlo simulation.

\subsection{Nonlinear multistory building under strong earthquake}

The following example is inspired from [28. A multistory shear-type building under a unilateral seismic excitation is considered. The structural model consists of $N_{s}$ stories modeled by lumped masses $m$ connected by clampedclamped nonlinear beam elements. The hardening behavior of a steel beam can be taken into account for large elastic displacements by an additional cubic nonlinear stiffness as in 24, 48. The structure is sketched in Fig. 4 and the undamped equation of motion for the $j$-th story is

$$
m \ddot{Y}_{j}+k\left(Y_{j+1}-2 Y_{j}+Y_{j-1}\right)+g_{j}\left(Y_{j-1}, Y_{j}, Y_{j+1}\right)=-m \ddot{U}
$$

with $g_{j}$ the $j$-th component of the nonlinear force vector defined as

$$
g_{j}\left(Y_{j-1}, Y_{j}, Y_{j+1}\right)=\kappa k\left(\left(Y_{j}-Y_{j-1}\right)^{3}-\left(Y_{j+1}-Y_{j}\right)^{3}\right)
$$

with $Y_{j}$ the relative horizontal displacement (with respect to the ground displacement) of that story. At the first floor, $Y_{0}$ is set equal to zero, while at the last floor, the term $Y_{N_{s}+1}-Y_{N_{s}}$ is also set equal to zero. In this illustration, the number of stories $N_{s}$ is fixed equal to 10 and the characteristics of the structure are $m=1290$ tons, $k=10^{8} \mathrm{~N} / \mathrm{m}$. The damping terms result from a proportional structural damping ratio imposed to $1 \%$ in the first two linear modes. The parameter $\kappa$ which quantifies the intensity of the nonlinear forces is supposed to be a variable parameter in this example. The natural frequencies of the first five linear normal modes are $0.21,0.62,1.02,1.40,1.75 \mathrm{~Hz}$. The unilateral excitation field is modeled by a modified Kanai-Tajimi spectrum [38]

$$
S_{\ddot{U}}=S_{0} \frac{\left(1+4 \xi_{1}^{2}\left(\frac{\omega}{\omega_{1}}\right)^{2}\right)}{\left(1-\left(\frac{\omega}{\omega_{1}}\right)^{2}\right)^{2}+4 \xi_{1}^{2}\left(\frac{\omega}{\omega_{1}}\right)^{2}} \frac{\left(\frac{\omega}{\omega_{2}}\right)^{4}}{\left(1-\left(\frac{\omega}{\omega_{2}}\right)^{2}\right)^{2}+4 \xi_{2}^{2}\left(\frac{\omega}{\omega_{2}}\right)^{2}}
$$


with $\omega_{1}=5 \mathrm{rad} / \mathrm{s}, \xi_{1}=0.2, \omega_{2}=0.5 \mathrm{rad} / \mathrm{s}, \xi_{2}=0.6$ and $S_{0}=0.03 \mathrm{~m}^{2} / \mathrm{s}^{3}$.

Since all the degrees of freedom are involved in the nonlinear behavior, this example is pertinent to illustrate the method. Furthermore, the classical fixed-point method might fail to solve this problem for moderate nonlinear behavior. Indeed, in the nodal basis $(n=10)$, for a given guess of $\boldsymbol{\Sigma}_{\mathbf{x}}^{(k)}$, the equivalent stiffness matrix $\mathbf{K}_{e q}$ may shift the natural frequencies in the high-frequency domain, for which the spectrum (59) is low. The updated response of the structure is therefore reduced, and so is the following guess of $\mathbf{K}_{e q}$. Thus, the natural frequencies are reduced and the risk here is to come back to the previous guess $\boldsymbol{\Sigma}_{\mathbf{x}}^{(k)}$. The fixed-point iterative process is thus eventually trapped in this period-2 oscillation, as illustrated in Fig. 4(b). This argument physically explains why the equation set is ill-conditioned and why the convergence can be really slow. Obviously, this fact corroborated by numerical experiments, shall not be encountered with white noise excitation processes. This might explain the attractivity of the fixed-point method, in more academic examples.
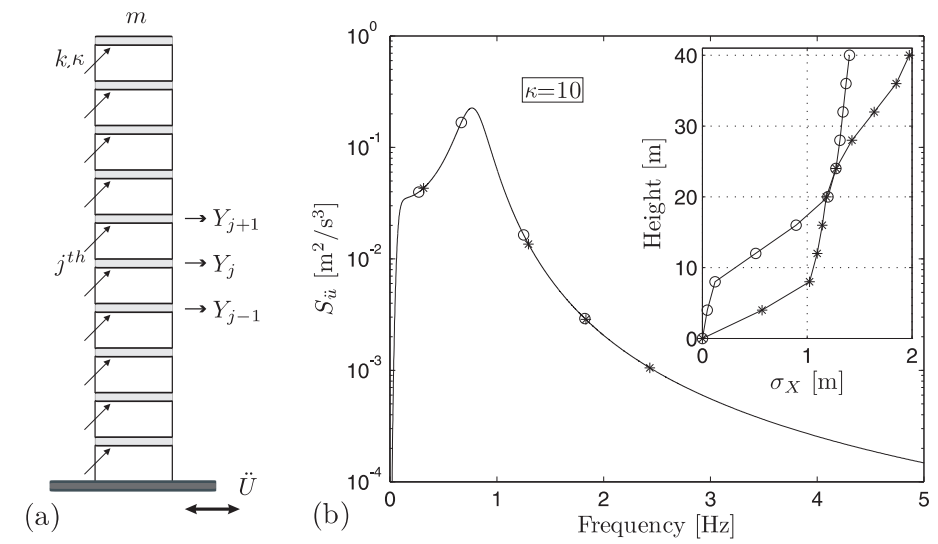

Figure 4: Sketch of the structure (a). Non-convergence of the fixed-point algorithm; Oscillations occur between the states $\circ$ and $*$, the standard deviations of the lateral displacements and the related natural frequencies on the spectrum are illustrated.

In this context, the proposed method (with $N=2$ ) shows its efficiency, because Newton's algorithm is able to explore more efficiently the solution space. With the proposed developments, the problem consists in minimizing the residue function (42), as well as keeping the convergence criterion (36) as small as possible. This latter aspect is ensured by a proper selection of the equivalent matrix $\tilde{\mathbf{K}}$. Due to the nonlinear stiffness forces, the initial choice of the matrix $\tilde{\mathbf{K}}$ for the modal projection in Equation 99 is important, because it conditions the convergence of the series. Computing $\mathcal{K}$ in (6) with $\mathbf{u}_{0}$ gives a crucial information about the distribution of the nonlinear forces and yet the amplitudes are roughly estimated. In this example, a good initial trial for $\tilde{\mathbf{K}}$ consists in computing $\mathcal{K}$ with a fraction of $\mathbf{u}_{0}$. This fraction can be interpreted as a ratio between the internal forces coming from the linear subsystem and from the equivalent linear one (in this problem the ratio $1 /(1+3 \kappa)$ is used). In this manner, both linear and equivalent linear forces are taken into account with an adequate proportion in the total stiffness matrix $\mathbf{K}+\tilde{\mathbf{K}}$. Although our goal is to keep it constant, matrix $\tilde{\mathbf{K}}$ may be updated, when a better estimate $\tilde{\mathbf{u}}$ is known $(\tilde{\mathbf{K}}=\mathcal{K}(\tilde{\mathbf{u}}))$ or when the convergence criterion is violated, in order to converge to a more accurate results. Here the proposed method reveals another valuable aspect, since the expansion-based method does not require a modal update at each iteration, as in a classical statistical linearization [10. In fact, examples show that one of two updates are by far enough to offer an accurate result. The combination of a small convergence criterion and a small residual function ensures that the solution is the sought one. 

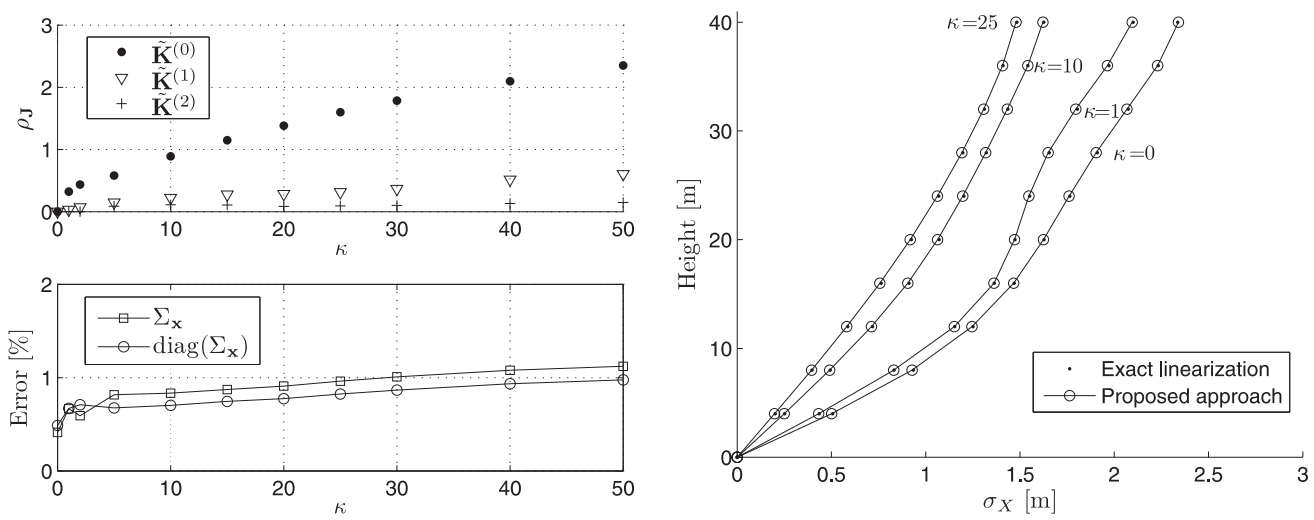

Figure 5: (a) Convergence criterion $\rho_{\mathbf{J}}$ for three iterations on $\tilde{\mathbf{K}}$. (b) Error [\%] between the proposed method $(N=2)$ and the formal statistical linearization. (c) Standard deviation of the lateral displacements for different values of $\kappa$.

For all simulations, five modes are kept in the analysis and $\tilde{\mathbf{K}}$ is updated twice in order to satisfy the convergence criterion as illustrated in Fig. 5(a). Even if $\rho_{\mathbf{J}}$ is greater than one with the first guess $\tilde{\mathbf{K}}^{(0)}$, it does not mean that the problem cannot be solved, but it shows that the solver does not converge to the good solution because the approximation of $\mathbf{s}(\mathbf{u})$ in $(42)$ is not accurate enough. However, the developed strategy just requires few iterations on $\tilde{\mathbf{K}}$ before reaching a suitable guess, as illustrated in Fig. 5(b), for strong nonlinarities. The comparison between Fig. 5(a) and (b) shows that a small index $\rho_{\mathbf{J}}$ (with a small residue) is a proof of convergence for the linearization. The nonlinear forces become more important, but a small index $\rho_{\mathbf{J}}$ ensures the converge independently of a value of $\kappa$. Figure (5)-c highlights the effects of the nonlinear forces on the standard deviation of the lateral displacements.

The number of iterations inside Newton's solver for all the simulations in Fig. 5 does not exceed four and the computation time does not exceed 1s per iterate (in MatLab): the method is not expensively consumming in virtue of its second-order accuracy. Figure 5(a) shows the accuracy of the method. The error on the diagonal terms and on the whole covariance matrix is at most 1\% even for strong nonlinearities. Figure 5(c) shows profiles of the standard deviation of the lateral displacements for different values of $\kappa$. The good agreement with the proposed method and the exact linearization is noteworthy.

\subsection{Response of a long-span bridge to a coherent seismic excitation}

As a third example, a conceptual 16-span bridge excited by a coherent seismic field is considered. Some connections between the piles and the deck are completed by nonlinear damping devices providing the isolation of the deck and the mitigation of the vibrations. The unilateral excitation $S_{i i}$ is modeled by a modified Kanai-Tajimi spectrum as in Equation (59) with the same parameters and the coherent seismic field $\mathbf{S}_{\ddot{\mathbf{u}}}(\omega)$ is modeled as

$$
\left(\mathbf{S}_{\ddot{\mathbf{u}}}\right)_{i j}=S_{\ddot{u}}\left|\Gamma_{i j}\right| \exp \left(\frac{-\iota \omega d_{i j}}{V}\right)
$$

with

$$
\left|\Gamma_{i j}\right|=A \exp \left(-\frac{2 d_{i j}}{\alpha \Theta_{s}}(1-A+\alpha A)\right)+(1-A) \exp \left(-\frac{2 d_{i j}}{\Theta_{s}}(1-A+\alpha A)\right)
$$

and $\Theta_{s}=k_{s}\left(1+\left|\frac{\omega}{\omega_{b}}\right|^{b}\right)^{-1 / 2}$, where $d_{i j}$ is the distance between the excitation points $i$ and $j$ The constants are chosen as $A=0.736, \alpha=0.147, k_{s}=512 \mathrm{~m}, b=2.78, \omega_{b}=6.85 \mathrm{rad} / \mathrm{s}$ and $V=600 \mathrm{~m} / \mathrm{s}$ [49, 50]. The structure is modeled with 3 -DOF beam finite elements $\left(210 \mathrm{GNm}^{2}, 6 \mathrm{t} / \mathrm{m}\right.$ for piles and deck) : the piles are $50 \mathrm{~m}$ height and the spans are $30 \mathrm{~m}$ long. The first natural frequencies of the linear substructure are $0.75,0.76,0.83,0.99$ and $1.25 \mathrm{~Hz}$ and the structural damping ratio is equal $1 \%$ for the first two modes. All the piles are horizontally connected to the deck by linear springs of $20 \mathrm{MN} / \mathrm{m}$ stiffness. On the connections at the piles 4, 6, 8, 10 and 12, nonlinear viscous dampers are set in order to mitigate the lateral vibrations in the first two modes of the structure (bending modes of 
the deck) during an earthquake. The force-velocity relationship of these devices is of the type $\eta \operatorname{sign}(v)|v|^{\beta}$ with $v$ the relative velocity between two connected DOF, $\eta$ the damping coefficient and $\beta$ ranged between 0.10 and 0.50 in order to be efficient for small velocity values. The stochastic linearization approach is commonly used to design such type of dampers [51]. Five modes are kept in the analysis and the modal basis is the one of the linear substructure $(\tilde{\mathbf{K}}=\mathbf{0})$.
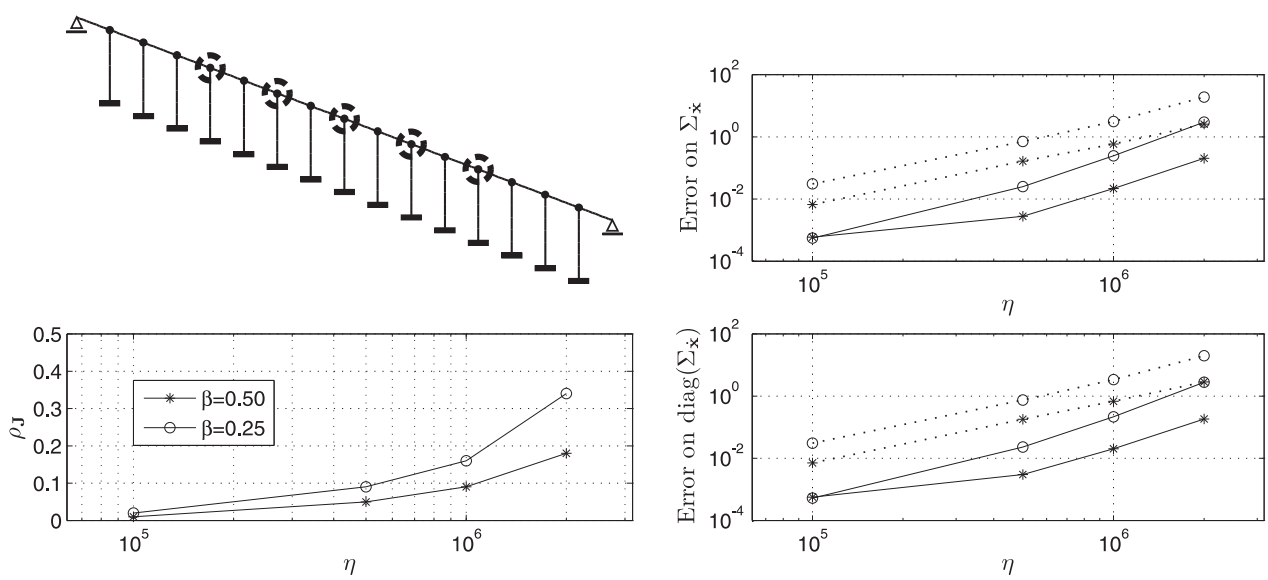

Figure 6: Sketch of the structure with the position of the dampers (a). Convergence criterion $\rho_{\mathbf{J}}$ for different values of $\eta$ (b). Error [\%] on the covariance matrix (c) and the variances (d) for different values of $\eta$ (dotted line $N=1$, solid line $N=2$ ).

Fig. 6(c) and (d) compare the equivalent linearization in a modal basis computed with a fixed-point approach and with the proposed expansion-based method (with $N=2$ ) for different combinations of $\beta$ and $\eta$. These results must be interpreted with regard to the convergence criterion $\rho_{\mathbf{J}}$ plotted in Fig. 6(b). For the case $\beta=0.50$, the index $\rho_{\mathbf{J}}$ does not exceed 0.15 , and the resulting errors are less than $1 \%$. However, for $\beta=0.25$, the nonlinear forces are stronger and the index $\rho_{\mathbf{J}}$ is thus greater than for $\beta=0.50$, for all the given values of $\eta$. In the case $\eta=2 \cdot 10^{6}$ and $\beta=0.25$, the maximum error is about $3 \%$ with $\rho_{\mathbf{J}}$ equal to 0.36 , which remains satisfactory. Furthermore, the fixed-point method has not converged and a continuation method has been applied in that case. The expansion-based method is able to deal with a large range of parameters in an efficient way. In Fig. 6, the variance of the lateral velocity of the deck are plotted in order to figure out the effect of the nonlinear viscous forces.
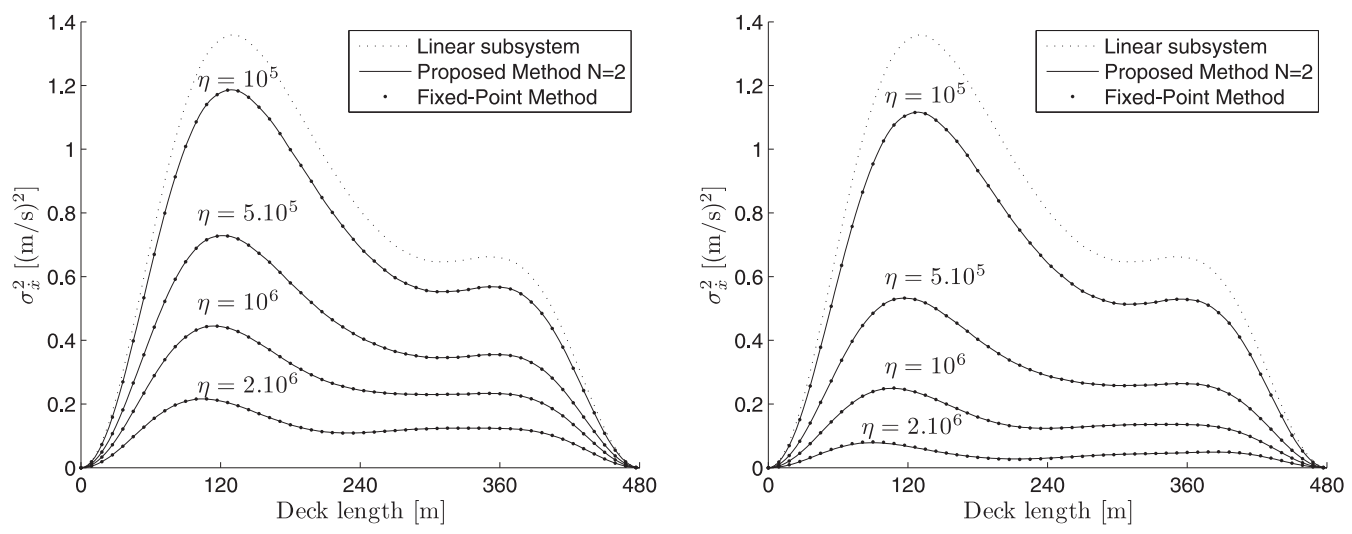

Figure 7: Variance of the lateral velocity of the deck for (a) $\beta=0.50$ and (b) $\beta=0.25$

The numerical efficiency is also checked between the proposed approach and the fixed-point approach. For each simulation, the initial guess is supposed to be the response of the linear subsystem. The asymptotic method converges to the linearized solution in spite of a huge difference with the initial guess. No more than 4 iterations are 
required in all cases to converge to the correct linearization with the proposed method. The number of iterations is divided by two, compared to the fixed-point approach (provided it converges).

\section{Conclusions}

The present paper has developed and applied the concept of asymptotic expansion of a modal transfer matrix in order to perform the GEL of large structures subject to coherent in space and time loadings. The proposed developments are based on the assumption that a suitable modal basis can be found to transform the equations of motion into a slightly coupled linearized system. According to some assumptions about the convergence of asymptotic expansion for matrices, the modal transfer matrix can be expanded in a series initiated by the transfer matrix of a main decoupled linearized system. Following linear algebra, inversion of full matrices is avoided and some remarkable integrals are extracted.

The choice of a suitable basis is a central discussion in this paper. With the asymptotic expansion-based method, a relevant basis must be able to transform the structural matrices into diagonally dominant matrices after the modal projection. While the use of a constant basis is limited to slightly nonlinear problems, moderately nonlinear problems may be treated with the proposed algorithms, with a very limited number of modal basis updates. As a major outcome, the projection of the nodal loading field, known as the most time-consuming operation in a spectral modal analysis, is performed only once (or only a couple of times depending on the amplitude of the nonlinearity) instead of each step, which increases the efficiency proportionally to the number of iterations.

These developments allow to use efficiently a Newton algorithm, i.e. a second-order method, in order to solve the nonlinear equation set involving integrals required to perform the linearization in a spectral strategy. Indeed, the computation of the Jacobian matrix is deeply simplified by the proposed approach. The combination of the asymptotic expansion-based method with this algorithm allows to use gradient-based method, quite efficiently.

Some examples are proposed in order to illustrate the robustness of the method, its ability to cover different natures and orders of magnitude for the nonlinear forces. Limitations of the proposed method with respect to the convergence criterion have also been illustrated. In particular, the expansion-based method remains competitive if the number of required correction terms is at most equal to two. We notice that two correction terms are mainly required, if $\rho_{\mathbf{J}}$ is about 0.4 . In all those cases, it is efficient and accurate at the same time.

\section{Acknowledgment}

The authors really appreciate the advices and suggestions expressed by the trustworthy friend, Alexandre Huynen. The first author would like to acknowledge the National Funds for Scientific Research of Belgium for its support. He also personally acknowledges Angelo Mariani for his kind attention and his true devotion. May he find in the completion of this work the fruit of his precious advices.

[1] H. Risken. The Fokker-Planck Equation. Methods of solution and applications. Springer-Verlag, Berlin, second edition edition, 1996.

[2] T. Canor and V. Denoël. Transient Fokker-Planck-Kolmogorov equation solved with Smoothed Particle Hydrodynamics. International Journal for Numerical Methods in Engineering, 94(6):535-553, 2013.

[3] D.P. Kroese, T. Taimre, and Z.I. Botev. Handbook of Monte Carlo Methods. Series in Probability and Statictics. Wiley, Hoboken, New-Jersey, 2011.

[4] C. Proppe, H. J. Pradlwarter, and G. I. Schuëller. Equivalent linearization and Monte-Carlo simulation in stochastic dynamics. Probabilistic Engineering Mechanics, 18(1):1-15, 2003.

[5] A. A. Taflanidis and G. Jia. A simulation-based framework for risk assessment and probabilistic sensitivity analysis of base-isolated structures. Earthquake Engineering 83 Structural Dynamics, 40(14):1629-1651, 2011.

[6] M. Shinozuka and G. Deodatis. Simulation of multi-dimensional Gaussian stochastic fields by spectral representation. Applied Mechanical Review (ASME), 49(1):29-53, 1996. 
[7] M. Di Paola. Digital simulation of wind field velocity. Journal of Wind Engineering and Industrial Aerodynamics, 74-76(0):91-109, 1998.

[8] M. Grigoriu. Stochastic Calculus. Applications in Science and Engineering. Birkhauser, Boston, 1st edition, 2002.

[9] L. Socha and T.T. Soong. Linearization in analysis of nonlinear stochastic systems. Applied Mechanics Review, 44(10):399-422, 1991.

[10] J.B. Roberts and P.D. Spanos. Random Vibration and Statistical Linearization. Dover Pub. Inc., Mineola (NY), 1st edition, 1999.

[11] P. Spanos, G. Failla, and M. Di Paola. Spectral approach to equivalent statistical quadratization and cubicization methods for nonlinear oscillators. Journal of Engineering Mathematics, 129(1):31-42, 2003.

[12] S.H. Crandall. Non-Gaussian closure techniques for stationary random vibration. International Journal of Non-Linear Mechanics, 20(1):1-8, 1985.

[13] T.K. Caughey. Response of a nonlinear string to random loading. Journal of Applied Mechanics (ASME), 26:341-344, 1959.

[14] T.K. Caughey. Response of van der Pol's oscillator to random loading. Journal of Applied Mechanics (ASME), 26:345-348, 1959.

[15] A.A.M Cunha. The role of the stochastic equivalent linearization method in the analysis of the non-linear seismic response of building structures. Earthquake Engineering and Structural Dynamics, 23(8):837-857, 1994.

[16] A. Y. J. Won, J. A. Pires, and M. A. Haroun. Stochastic seismic performance evaluation of tuned liquid column dampers. Earthquake Engineering and Structural Dynamics, 25(11):1259-1274, 1996.

[17] E. Esmaeilzadeh Seylabi, H. Jahankhah, and M. Ali Ghannad. Equivalent linearization of non-linear soilstructure systems. Earthquake Engineering \&3 Structural Dynamics, 41(13):1775-1792, 2012.

[18] Y. L. Xu, B. Samali, and K. C. S. Kwok. Control of along-wind response of structures by mass and liquid dampers. Journal of Engineering Mechanics, 118(1):20-39, 1992.

[19] T. K. Guha, R. N. Sharma, and P. J. Richards. Internal pressure in a building with multiple dominant openings in a single wall: Comparison with the single opening situation. Journal of Wind Engineering and Industrial Aerodynamics, 107-108(0):244-255, 2012.

[20] F. Gani and F. Légeron. Relationship between specified ductility and strength demand reduction for single degree-of-freedom systems under extreme wind events. Journal of Wind Engineering and Industrial Aerodynamics, 109(0):31-45, 2012.

[21] M. Grigoriu. Equivalent linearization for systems driven by Lévy white noise. Probabilistic Engineering Mechanics, 15(2):185-190, 2000.

[22] C. Proppe. Stochastic linearization of dynamical systems under parametric Poisson white noise excitation. International Journal of Non-Linear Mechanics, 38(4):543-555, 2003.

[23] S.H. Crandall. On using non-Gaussian distributions to perform statistical linearization. International Journal of Non-Linear Mechanics, 39(9):1395-1406, 2004.

[24] G. Ricciardi. A non-Gaussian stochastic linearization method. Probabilistic Engineering Mechanics, 22(1):1-11, 2007.

[25] V. Denoël and H. Degée. Asymptotic expansion of slightly coupled modal dynamic transfer functions. Journal of Sound and Vibration, 328:1-8, 2009. 
[26] T. Canor, N. Blaise, and V. Denoël. Efficient uncoupled stochastic analysis with non-propotional damping. Journal of Sound and Vibration, 331:5283-5291, 2012.

[27] M. B. Priestley. Evolutionary spectra and non-stationary processes. Journal of the Royal Statistical Society. Series B (Methodological), 27(2):204-237, 1965.

[28] Y.K. Lin and G.Q. Cai. Probabilistic Structural Dynamics. Advanced Theory and Applications. McGraw-Hill, New-York, 2nd edition, 2004.

[29] S. Rezaeian and A. der Kiureghian. A stochastic ground motion model with separable temporal and spectral nonstationarities. Earthquake Engineering 83 Structural Dynamics, 37(13):1565-1584, 2008.

[30] J. K. Hammond. Evolutionary spectra in random vibrations. Journal of the Royal Statistical Society. Series B (Methodological), 35(2):167-188, 1973.

[31] I.A. Kougioumtzoglou and P.D. Spanos. Nonlinear MDOF system stochastic response determination via a dimension reduction approach. Computers \& Structures, 126:135-148, 2013.

[32] R. H. Bartels and G. W. Stewart. Solution of the matrix equation $\mathrm{AX}+\mathrm{XB}=\mathrm{C}$. Communications of the ACM, 15(9):820-826, 1972.

[33] F. L. Silva and S. E. Ruiz. Calibration of the equivalent linearization Gaussian approach applied to simple hysteretic systems subjected to narrow band seismic motions. Structural Safety, 22(3):211-231, 2000.

[34] D. C. Sorensen and Zhou. Direct methods for matrix Sylvester and Lyapunov equations. Journal of Applied Mathematics, 6(2003):277-303, 2003.

[35] A. Quarteroni, R. Sacco, and F. Saleri. Numerical Mathematics, volume 1 of Texts in Applied Mathematics. Springer Verlag, Berlin, 2nd edition edition, 2007.

[36] E. Simiu and R. Scanlan. Wind Effects On Structures. John Wiley and Sons, 3rd edition, 1996.

[37] A. Der Kiureghian and A. Neuenhofer. Response spectrum method for multi-support seismic excitations. Earthquake Engineering and Structural Dynamics, 21(8):713-740, 1992.

[38] R. W. Clough and J. Penzien. Dynamics of structures. McGraw-Hill, New-York, 2nd edition, 1993.

[39] H.J. Pradlwarter, G.I. Schuëller, and C.A. Schenk. A computational procedure to estimate the stochastic dynamic response of large non-linear FE-models. Computer Methods in Applied Mechanics and Engineering, 192:777-801, 2003.

[40] R.A. Horn and Johnson C.R. Matrix Analysis. Cambridge University Press, Cambridge, seconde edition edition, 2013.

[41] M. Morzfeld, N. Ajavakom, and F. Ma. Diagonal dominance of damping and the decoupling approximation in linear vibratory systems. Journal of Sound and Vibration, 320:406-420, 2009.

[42] W.H. Press, S.A. Teukolsky, W.T. Vetterling, and B.P. Flannery. Numerical Recipes. The art of scientific computing. Cambridge University Press, 3rd edition, 2007.

[43] E. Kreyszig. Advanced Engineering Mathematics. John Wiley and Sons, Inc., 9th edition, 2006.

[44] A. G. Davenport. The application of statistical concepts to the wind loading of structures. Proceedings of the Institute of Civil Engineers, 19:449-472, 1961.

[45] V. Denoël. Estimation of modal correlation coefficients from background and resonant responses. Structural Engineering and Mechanics, 32(6), 2009. 
[46] F Sakai, S Takaeda, and T Tamaki. Tuned liquid column damper-new type device for suppression of building vibrations. In Proceedings of International Conference on Highrise Buildings, Nanjing, China, volume 926, page 931, 1989.

[47] Y. Saoka, F. Sakai, S. Takaeda, and T. Tamaki. On the suppression of vibrations by tuned liquid column dampers. In Annual Meeting of JSCE, JSCE, Tokyo, 1988.

[48] X. L. Jin, Z. L. Huang, and A. Y. T. Leung. Nonstationary seismic responses of structure with nonlinear stiffness subject to modulated Kanai-Tajimi excitation. Earthquake Engineering \& Structural Dynamics, 41(2):197-210, 2012 .

[49] R. S. Harichandran and E. H. Vanmarcke. Stochastic variation of earthquake ground motion in space and time. Journal of Engineering Mechanics, 112(2):154-174, 1986.

[50] R. S. Harichandran, A. Hawwari, and B. N. Sweidan. Response of long-span bridges to spatially varying ground motion a. Journal of Structural Engineering, 122(5):476-484, 1996.

[51] M. Paola, L. Mendola, and G. Navarra. Stochastic seismic analysis of structures with nonlinear viscous dampers. Journal of Structural Engineering, 133(10):1475-1478, 2007.

\section{AppendixA. A sufficient condition of convergence of the asymptotic expansion}

Invoking the Gerschgorin theorem [40, a subspace $\mathcal{B}(\mathbf{A})$ of $\mathbb{C}$ containing all the eigenvalues of a matrix $\mathbf{A}=$ $\left[a_{i j}\right] \in \mathbb{C}^{m \times m}$ can be defined. This subspace is the union of the so-called Gerschgorin discs defined as $D_{i}\left(a_{i i}, R_{i}\right)$ with the radius $R_{i}=\sum_{j=1, j \neq 1}^{m}\left|a_{i j}\right|$ and $a_{i i}$ the center of the circle. Thus, $\mathcal{B}(\mathbf{A})=\bigcup_{i=1}^{m} D_{i}\left(a_{i i}, R_{i}\right)$ and the $i$-th eigenvalue $\lambda_{\mathbf{A}, i} \in \mathcal{B}(\mathbf{A})$ with $i=1, \ldots m$. If $\mathbf{A}$ is posed equal to $\mathbf{H}_{d}(\omega) \mathbf{J}_{o}(\omega)$ as in Equation $(20)$, the theorem reads

$$
D_{i}=\left\{z \in \mathbb{C}, \omega \in \mathbb{R},|z| \leq \sum_{j \neq i=1}^{n}\left|\left(\mathbf{H}_{d}\right)_{i}\left(\mathbf{J}_{o}\right)_{i j}\right|\right\},
$$

since the diagonal elements of $\mathbf{H}_{d} \mathbf{J}_{o}$ are equal to zero. Therefore, the $m$ Gerschgorin discs are concentric. The inequality defining the $i$-th disc can be rewritten and an upper bound is calculated as

$$
\begin{aligned}
|z| & \leq\left|\left(\mathbf{H}_{d}(\omega)\right)_{i}\right| \sum_{j \neq i=1}^{n} \sqrt{\left(\boldsymbol{\Omega}_{o}\right)_{i j}^{2}+\omega^{2}\left(\mathbf{D}_{o}\right)_{i j}^{2}} \\
& <\left|\left(\mathbf{H}_{d}\left(\omega_{d, i}\right)\right)_{i}\right| \sum_{j \neq i=1}^{n} \sqrt{\left(\boldsymbol{\Omega}_{o}\right)_{i j}^{2}+\omega_{d, i}^{2}\left(\mathbf{D}_{o}\right)_{i j}^{2}}
\end{aligned}
$$

with $\omega_{d, i}$ defined in Section 4.3 . Notice that no formal distinction is introduced between natural frequency and the damped natural frequency of the decoupled equivalent system.

\section{AppendixB. Extension of the background-resonant approximation}

The background-resonant approximation introduced by Davenport in [44 is a common method in wind engineering used to compute the integral of the product between the PSD of a background excitation and the transfer function of a linear SDOF system in order to estimate the variance of the response. The philosophy of the method is based on the evident separation between two timescales in the process : (i) the quasi-static response of the system due to the low frequency excitation and (ii) the resonant response due to dynamical amplification of the system at higher frequency content. This methodology is applied here in order to facilitate the computation of the integral $\mathcal{I}_{i j k, \alpha}$, in presence of low frequency excitations like turbulent wind. 
First, the integral $\mathcal{I}_{i i i, 0}$ is considered. The background component is rapidly computed by adding and subtracting simultaneously a term $\left|\left(\mathbf{H}_{d}(0)\right)_{i}\right|^{3}$, such that

$$
\begin{aligned}
\int_{\mathbb{R}}\left(\mathbf{H}_{d}\right)_{i}\left(\mathbf{H}_{d}\right)_{i}\left(\mathbf{S}_{\mathbf{p}}\right)_{i i}\left(\mathbf{H}_{d}\right)_{i}^{*} d \omega & =\frac{1}{\omega_{0, i}^{6}} \int_{\mathbb{R}}\left(\mathbf{S}_{\mathbf{p}}\right)_{i i} d \omega+\int_{\mathbb{R}}\left(\left(\mathbf{H}_{d}\right)_{i}\left(\mathbf{H}_{d}\right)_{i}\left(\mathbf{H}_{d}\right)_{i}^{*}-\frac{1}{\omega_{0, i}^{6}}\right)\left(\mathbf{S}_{\mathbf{p}}\right)_{i i} d \omega \\
& =\frac{1}{\omega_{0, i}^{6}}\left(\boldsymbol{\Sigma}_{\mathbf{p}}\right)_{i i}+\int_{\mathbb{R}} \phi(\omega)\left(\mathbf{S}_{\mathbf{p}}\right)_{i i} d \omega,
\end{aligned}
$$

where $\boldsymbol{\Sigma}_{\mathbf{p}}$ is the covariance matrix of the modal forces. The first term is the background contribution, while the second integral is the resonant one. Two identical and symmetric contributions to the resonant component are concentrated around the natural frequency $\pm \omega_{0, i}$, so just one of those is computed for $\omega>0$. A coordinate $\eta$ is used to stretch the frequency $\omega$ around $\omega_{0, i}$, such that $\omega=\omega_{0, i}(1+\xi \eta)$. The previous integral now reads

$$
\int_{\mathbb{R}} \phi(\omega)\left(\mathbf{S}_{\mathbf{p}}\right)_{i i} d \omega \approx 2 \xi \omega_{0, i} \int_{\mathbb{R}^{+}} \phi(\eta)\left(\mathbf{S}_{\mathbf{p}}\right)_{i i} d \eta
$$

However, the function $\phi(\eta)$ remains non-integrable on $+\infty$, so that function is replaced by a local Padé approximation $\tilde{\phi}(\eta)$ of the real part of $\phi(\eta)$, since the integral is real-valued. Everywhere else, the Padé approximation $\tilde{\phi}(\eta)$ is continuous and tends to zero for $\eta$ tending to $0,+\infty$ and $-\infty$. The resonant contribution is thus approximated by

$$
\int_{\mathbb{R}} \phi(\omega)\left(\mathbf{S}_{\mathbf{p}}\right)_{i i} d \omega \approx 2 \int_{\mathbb{R}} \tilde{\phi}(\eta)\left(\mathbf{S}_{\mathbf{p}}\right)_{i i} \xi \omega_{0, i} d \eta .
$$

Locally, the spectrum $\mathbf{S}_{\mathbf{p}}(\omega)$ is approximated by its first-order expansion and the integral becomes

$$
\int_{\mathbb{R}} \phi(\omega)\left(\mathbf{S}_{\mathbf{p}}\right)_{i i} d \omega \approx 2 \int_{\mathbb{R}} \tilde{\phi}(\eta)\left(\left(\mathbf{S}_{\mathbf{p}}\left(\omega_{0, i}\right)\right)_{i i}+\eta \xi \omega_{0, i}\left(\mathbf{S}_{\mathbf{p}}^{\prime}\left(\omega_{0, i}\right)\right)_{i i}\right) \xi \omega_{0, i} d \eta
$$

The remaining integrals can be performed with the residue theorem. For $\alpha>0$, the integral $\mathcal{I}_{i i i, \alpha}$ has no background contribution. The methodology is applied also to these integrals in order to obtained simplified formula. Some pertinent results are

$$
\mathcal{I}_{i i i, 1}=\frac{\pi}{8 \xi_{i}^{2} \omega_{d, i}^{4}}\left(\mathbf{S}_{\mathbf{p}}\left(\omega_{d, i}\right)\right)_{i i}, \quad \mathcal{I}_{i i i, 2}=\frac{\pi}{8 \xi_{i} \omega_{d, i}^{2}}\left(\mathbf{S}_{\mathbf{p}}^{\prime}\left(\omega_{d, i}\right)\right)_{i i}, \quad \mathcal{I}_{i i i, 3}=\frac{-\pi}{8 \xi_{i}^{2} \omega_{d, i}^{2}}\left(\mathbf{S}_{\mathbf{p}}\left(\omega_{d, i}\right)\right)_{i i} .
$$

